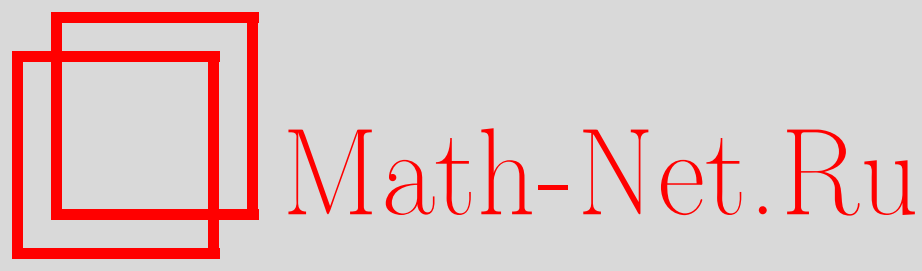

А. Г. Смирнов, О разложениях по собственным функциям для уравнения Шредингера с обратноквадратичным потенциалом, ТМФ, 2016, том 187, номер 2, 360-382

DOI: https://doi.org/10.4213/tmf9032

Использование Общероссийского математического портала Math-Net.Ru подразумевает, что вы прочитали и согласны с пользовательским соглашением http://www . mathnet.ru/rus/agreement

Параметры загрузки:

IP: 54.81 .137 .203

26 апреля 2023 г., 16:21:20

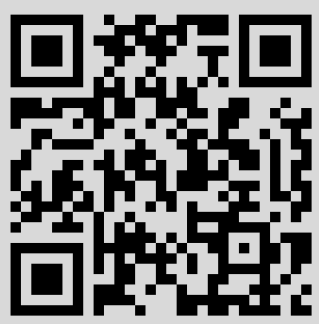




\title{
ФИЗИКА
}

Том 187, № 2

май, 2016

(C) 2016 г.

А. Г. Смирнов*

\section{О РАЗЛОЖЕНИЯХ ПО СОБСТВЕННЫМ ФУНКЦИЯМ ДЛЯ УРАВНЕНИЯ ШРЕДИНГЕРА С ОБРАТНОКВАДРАТИЧНЫМ ПОТЕНЦИАЛОМ}

\begin{abstract}
Рассмотрено одномерное уравнение Шредингера $-f^{\prime \prime}+q_{\kappa} f=E f$ на положительной полуоси с потенциалом $q_{\kappa}(r)=\left(\kappa^{2}-1 / 4\right) r^{-2}$. Для каждого комплексного $\vartheta$ построено решение $u_{\vartheta}^{\kappa}(E)$ данного уравнения, аналитическое по $\kappa$ в комплексной окрестности интервала $(-1,1)$ и, в частности, в "сингулярной" точке $\kappa=0$. При $-1<\kappa<1$ и вещественных $\vartheta$ решения $u_{\vartheta}^{\kappa}(E)$ однозначно определяют унитарный оператор разложения по собственным функциям $U_{\kappa, \vartheta}: L_{2}(0, \infty) \rightarrow L_{2}\left(\mathbb{R}, \mathcal{V}_{\kappa, \vartheta}\right)$, где $\mathcal{V}_{\kappa, \vartheta}$ - положительная мера на пространстве $\mathbb{R}$. Показано, что каждая самосопряженная реализация формального дифференциального выражения $-\partial_{r}^{2}+q_{\kappa}(r)$ для гамильтониана диагонализуется оператором $U_{\kappa, \vartheta}$ при некотором $\vartheta \in \mathbb{R}$. При помощи подходящих сингулярных $m$-функций Титчмарша-Вейля явно найдены меры $\mathcal{V}_{\kappa, \vartheta}$ и доказана их непрерывность по $\kappa$ и $\vartheta$.
\end{abstract}

Ключевые слова: уравнение Шредингера, обратноквадратичный потенциал, самосопряженные расширения, разложения по собственным функциям, $m$-функция Титчмарша-Вейля.

DOI: $10.4213 / \operatorname{tmf9032}$

Посвящается профессору И.В. Тютину в связи с его 75-летием

\section{1. ВВЕДЕНИЕ}

Настоящая работа посвящена разложениям по собственным функциям, связанным с одномерным уравнением Шредингера

$$
-\partial_{r}^{2} f(r)+\frac{\kappa^{2}-1 / 4}{r^{2}} f(r)=E f(r), \quad r>0
$$

где $\kappa$ и $E$ - вещественные параметры. Нетрудно видеть, что функция $f(r)=$ $r^{1 / 2} J_{\kappa}\left(E^{1 / 2} r\right)$, где $J_{\kappa}$ - функция Бесселя первого рода порядка $\kappa$, представляет собой решение уравнения (1) для любых $E>0$ и $\kappa \in \mathbb{R}$ (это немедленно следует из

* Физический институт им. П. Н. Лебедева РАН, Москва, Россия.

E-mail: smirnov@lpi.ru 
того, что $J_{\kappa}$ удовлетворяет уравнению Бесселя). Эти решения можно использовать для разложения квадратично-интегрируемых функций на положительной полуоси $\mathbb{R}_{+}=(0, \infty)$. Точнее говоря, если имеются $\kappa>-1$ и квадратично-интегрируемая комплексная функция $\psi$ на $\mathbb{R}_{+}$, обращающаяся в нуль при больших $r$, то мы можем определить функцию $\hat{\psi}$ на $\mathbb{R}_{+}$при помощи соотношения

$$
\hat{\psi}(E)=\frac{1}{\sqrt{2}} \int_{0}^{\infty} \sqrt{r} J_{\kappa}(\sqrt{E} r) \psi(r) d r, \quad E>0 .
$$

Тогда отображение $\psi \rightarrow \hat{\psi}$ с точностью до замены переменных совпадает с известным преобразованием Ханкеля [1] и однозначно задает унитарный оператор в $L_{2}\left(\mathbb{R}_{+}\right)$. С тех пор как Вейлем [2] была развита общая теория сингулярных задач Штурма-Лиувилля, данное преобразование использовалось многими авторами для иллюстрации различных подходов к разложениям по собственным функциям в такого рода задачах [3]-[8].

Если $\kappa \geqslant 1$, то преобразование (2) с точностью до нормировки собственных функций является единственным разложением по собственным функциям, которое можно связать с уравнением (1). С другой стороны, при $|\kappa|<1$ из решений уравнения (1) можно построить однопараметрическое семейство различных разложений (см. главу 4 в монографии [4]). Причина такой неоднозначности заключается в том, что формальное дифференциальное выражение для гамильтониана

$$
-\partial_{r}^{2}+\frac{\kappa^{2}-1 / 4}{r^{2}}
$$

при $|\kappa|<1$ не определяет квантово-механическую задачу единственным образом и допускает различные самосопряженные реализации в пространстве $L_{2}\left(\mathbb{R}_{+}\right)$, которым отвечают различные разложения по собственным функциям. В работе [9] все самосопряженные реализации выражения (3) были охарактеризованы при помощи подходящих асимптотических граничных условий и были явно найдены соответствующие разложения по собственным функциям.

Как в [4], так и в [9] случаи $0<|\kappa|<1$ и $\kappa=0$ рассматривались по отдельности, причем разложения по собственным функциям при $\kappa=0$ не могли быть получены из разложений при $0<|\kappa|<1$ при помощи предельного перехода $\kappa \rightarrow 0$. Такая ситуация не вполне удовлетворительна с физической точки зрения. Самосопряженные операторы, связанные с выражением (3), можно использовать, в том числе, для построения самосопряженных реализаций гамильтониана Ааронова-Бома [10]. При этом $\kappa$, равное нулю, и $\kappa$, отличные от нуля, отвечают соответственно целым и нецелым значениям безразмерного магнитного потока сквозь соленоид. Поэтому существование хорошо определенного предела $\kappa \rightarrow 0$ необходимо для того, чтобы иметь непрерывный переход между целыми и нецелыми значениями потока в модели Ааронова-Бома. В данной статье предлагается параметризация самосопряженных реализаций выражения (3) и соответствующих разложений по собственным функциям, обладающая свойством непрерывности по $\kappa$ на интервале $(-1,1)($ и, в частности, при $\kappa=0)$.

Сформулируем теперь основные результаты статьи. Пусть $\lambda$ - мера Лебега на $\mathbb{R}$, а $C_{0}^{\infty}\left(\mathbb{R}_{+}\right)$- пространство всех гладких функций на $\mathbb{R}_{+}$с компактным носителем. 
Если имеется функция $f$, определенная $\lambda$-п.в. ${ }^{1)}$ на $\mathbb{R}_{+}$, то ее класс эквивалентности относительно меры Лебега на $\mathbb{R}_{+}$(т. е. сужения меры $\lambda$ на $\mathbb{R}_{+}$) обозначим через $[f]$. Для каждого $\kappa \in \mathbb{R}$ дифференциальное выражение (3) естественным образом определяет оператор $\check{h}_{\kappa}$ в $L_{2}\left(\mathbb{R}_{+}\right)$, область определения $D_{\check{h}_{\kappa}}$ которого состоит из всех элементов $[f]$, отвечающих $f \in C_{0}^{\infty}\left(\mathbb{R}_{+}\right)$:

$$
\begin{aligned}
D_{\breve{h}_{\kappa}} & =\left\{[f]: f \in C_{0}^{\infty}\left(\mathbb{R}_{+}\right)\right\}, \\
\check{h}_{\kappa}[f] & =\left[-f^{\prime \prime}+q_{\kappa} f\right], \quad f \in C_{0}^{\infty}\left(\mathbb{R}_{+}\right) .
\end{aligned}
$$

Через $q_{\kappa}$ здесь обозначен потенциальный член в (3):

$$
q_{\kappa}(r)=\frac{\kappa^{2}-1 / 4}{r^{2}}, \quad r \in \mathbb{R}_{+} .
$$

Оператор $\check{h}_{\kappa}$, очевидно, является симметрическим и, следовательно, замыкаем. Замыкание $\breve{h}_{\kappa}$ будем обозначать через $h_{\kappa}$ :

$$
h_{\kappa}=\check{\check{h}_{\kappa}} .
$$

Самосопряженные расширения $h_{\kappa}$ (или, что то же самое, $\check{h}_{\kappa}$ ) естественно понимать как самосопряженные реализации формального выражения (3) (ср. замечание 5 ниже).

Для любых $z, \kappa \in \mathbb{C}$ определим функцию $u^{\kappa}(z)$ на $\mathbb{R}_{+}$посредством соотношения ${ }^{2)}$

$$
u^{\kappa}(z \mid r)=r^{1 / 2+\kappa} \mathcal{X}_{\kappa}\left(r^{2} z\right), \quad r \in \mathbb{R}_{+},
$$

где целая функция $\mathcal{X}_{\kappa}$ дается формулой

$$
\mathcal{X}_{\kappa}(\zeta)=\frac{1}{2^{\kappa}} \sum_{n=0}^{\infty} \frac{(-1)^{n} \zeta^{n}}{\Gamma(\kappa+n+1) n ! 2^{2 n}}, \quad \zeta \in \mathbb{C} .
$$

Функция $\mathcal{X}_{\kappa}$ тесно связана с функциями Бесселя: при $z \neq 0$ справедливо равенство

$$
\mathcal{X}_{\kappa}(\zeta)=\zeta^{-\kappa / 2} J_{\kappa}\left(\zeta^{1 / 2}\right)
$$

Поскольку $J_{\kappa}$ удовлетворяет уравнению Бесселя, имеем

$$
-\partial_{r}^{2} u^{ \pm \kappa}(z \mid r)+q_{\kappa}(r) u^{ \pm \kappa}(z \mid r)=z u^{ \pm \kappa}(z \mid r), \quad r \in \mathbb{R}_{+},
$$

для всех $\kappa \in \mathbb{C}$ и $z \neq 0^{3)}$. По непрерывности это верно и при $z=0$. В частности, $u^{ \pm \kappa}(E)$ являются решениями спектральной задачи (1) для любых $\kappa, E \in \mathbb{R}$.

Если имеются положительная борелевская мера $\sigma$ на $\mathbb{R}$ и $\sigma$-измеримая комплексная функция $g$, то обозначим через $\mathcal{T}_{g}^{\sigma}$ оператор умножения на $g$ в $L_{2}(\mathbb{R}, \sigma)^{4)}$. Если

\footnotetext{
1) Мы используем здесь общепринятое сокращение п.в., которое означает либо "почти все", либо "почти всюду".

2) Для краткости через $u^{\kappa}(z \mid r)$ будем обозначать значение функции $u^{\kappa}(z)$ в точке $r: u^{\kappa}(z \mid r)=$ $\left(u^{\kappa}(z)\right)(r)$.

3) Здесь и далее предполагается, что функция $q_{\kappa}$ на $\mathbb{R}_{+}$определена формулой $(5)$ для всех $\kappa \in \mathbb{C}$.

4) Точнее говоря, $\mathcal{T}_{g}^{\sigma}$ есть оператор в $L_{2}(\mathbb{R}, \sigma)$, график которого состоит из всех таких пар $\left(\varphi_{1}, \varphi_{2}\right)$, что $\varphi_{1}, \varphi_{2} \in L_{2}(\mathbb{R}, \sigma)$ и $\varphi_{2}(E)=g(E) \varphi_{1}(E)$ для $\sigma$-п.в. $E$.
} 
функция $g$ вещественна, то оператор $\mathcal{T}_{g}^{\sigma}$ самосопряжен. При $\kappa>-1$ определим положительную меру Радона ${ }^{5)} \mathcal{V}_{\kappa}$ на $\mathbb{R}$ посредством соотношения

$$
d \mathcal{V}_{\kappa}(E)=\frac{1}{2} \Theta(E) E^{\kappa} d E
$$

где $\Theta$ - функция Хевисайда, т. е. $\Theta(E)=1$ при $E \geqslant 0$ и $\Theta(E)=0$ при $E<0$. Обозначим через $L_{2}^{c}\left(\mathbb{R}_{+}\right)$подпространство пространства $L_{2}\left(\mathbb{R}_{+}\right)$, состоящее из всех его элементов, обращающихся в нуль $\lambda$-п.в. вне некоторого компактного подмножества $\mathbb{R}_{+}$.

Известно (см., например, [5], [6], [9]), что при $\kappa \geqslant 1$ оператор $h_{\kappa}$ самосопряжен и может быть диагонализован при помощи преобразования Ханкеля (2). В терминах функций $u^{\kappa}(z)$ этот результат можно сформулировать следующим образом.

Теорема 1. Пусть $\kappa>-1$ и мера $\mathcal{V}_{\kappa}$ на $\mathbb{R}$ определена формулой (11). Тогда существует единственный унитарный оператор $U_{\kappa}: L_{2}\left(\mathbb{R}_{+}\right) \rightarrow L_{2}\left(\mathbb{R}, \mathcal{V}_{\kappa}\right)$ такой, что

$$
\left(U_{\kappa} \psi\right)(E)=\int_{0}^{\infty} u^{\kappa}(E \mid r) \psi(r) d r, \quad \psi \in L_{2}^{c}\left(\mathbb{R}_{+}\right),
$$

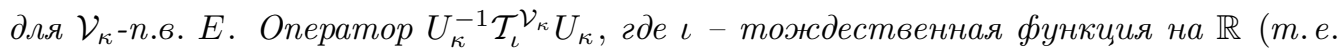
$\iota(E)=E$ для всех $E \in \mathbb{R})$, есть самосопряженное расширение $h_{\kappa}$, совпадающее $с h_{\kappa}$ при $\kappa \geqslant 1$.

В силу (7) и (9) имеем $u^{\kappa}(E \mid r)=E^{-\kappa / 2} r^{1 / 2} J_{\kappa}\left(E^{1 / 2} r\right), r \in \mathbb{R}_{+}$, для любого $E>0$. Поэтому оператор $U_{\kappa}$ совпадает с преобразованием $(2)$ с точностью до нормировки собственных функций. Заметим, что $h_{\kappa}=h_{|\kappa|}$ для всех $\kappa \in \mathbb{R}$, и, следовательно, $h_{\kappa}$ диагонализуется оператором $U_{|\kappa|}$ для всех таких вещественных $\kappa$, что $|\kappa| \geqslant 1$. Если $0 \leqslant \kappa<1$, то $U_{\kappa}^{-1} \mathcal{T}_{\iota} \mathcal{V}_{\kappa} U_{\kappa}$ представляет собой расширение Фридрихса $h_{\kappa}$ (см. [11]).

Обратимся теперь к параметризации всех самосопряженных расширений $h_{\kappa}$ в случае $-1<\kappa<1$. Пусть

$$
\mathcal{O}=\{\kappa \in \mathbb{C}: \kappa \neq \pm 1, \pm 2, \ldots\}
$$

Для $\kappa \in \mathcal{O}$ и $\vartheta, z \in \mathbb{C}$ определим функцию $u_{\vartheta}^{\kappa}(z)$ на $\mathbb{R}_{+}$, полагая

$$
u_{\vartheta}^{\kappa}(z)=\frac{u^{\kappa}(z) \sin \left(\vartheta+\vartheta_{\kappa}\right)-u^{-\kappa}(z) \sin \left(\vartheta-\vartheta_{\kappa}\right)}{\sin \pi \kappa}, \quad \kappa \in \mathcal{O} \backslash\{0\},
$$

И

$$
\begin{aligned}
& u_{\vartheta}^{0}(z \mid r)=\lim _{\kappa \rightarrow 0} u_{\vartheta}^{\kappa}(z \mid r)= \\
& =u^{0}(z \mid r) \cos \vartheta+\frac{2}{\pi}\left[\left(\ln \frac{r}{2}+\gamma\right) u^{0}(z \mid r)-\sqrt{r} \mathcal{Y}\left(r^{2} z\right)\right] \sin \vartheta, \quad r \in \mathbb{R}_{+},
\end{aligned}
$$

где

$$
\vartheta_{\kappa}=\frac{\pi \kappa}{2}
$$

${ }^{5)}$ Напомним, что борелевская мера $\sigma$ на $\mathbb{R}$ называется мерой Радона на $\mathbb{R}$, если $\sigma(K)<\infty$ для любого компактного множества $K \subset \mathbb{R}$. 
целая функция $\mathcal{Y}$ дается формулой

$$
\mathcal{Y}(\zeta)=\sum_{n=1}^{\infty} \frac{(-1)^{n} c_{n}}{(n !)^{2} 2^{2 n}} \zeta^{n}, \quad c_{n}=\sum_{j=1}^{n} \frac{1}{j},
$$

и $\gamma=\lim _{n \rightarrow \infty}\left(c_{n}-\ln n\right)=0,577 \ldots$ - константа Эйлера ${ }^{6)}$.

Для $\alpha \in \mathbb{R}$ положим $R_{\alpha}=\left\{z \in \mathbb{C}: z=r e^{i \alpha}\right.$ для некоторого $\left.r \geqslant 0\right\}$ и

$$
\mathbb{C}_{\alpha}=\mathbb{C} \backslash R_{\alpha} .
$$

Таким образом, $\mathbb{C}_{\alpha}$ есть комплексная плоскость с разрезом вдоль луча $R_{\alpha}$.

Следующее утверждение показывает, что величина $u_{\vartheta}^{\kappa}(z \mid r)$, несмотря на кусочный характер ее определения, в действительности аналитична по всем своим аргументам.

Лемма 2. Существует единственная функция $F$, аналитическая в области $\mathcal{O} \times \mathbb{C} \times \mathbb{C} \times \mathbb{C}_{\pi}$ и удовлетворяющая равенству $F(\kappa, \vartheta, z, r)=u_{\vartheta}^{\kappa}(z \mid r)$ для любъх $\vartheta, z \in \mathbb{C}, \kappa \in \mathcal{O}$ ur $\in \mathbb{R}_{+}$.

Доказательство леммы 2 приведено в приложении А.

Для любых $\kappa \in \mathcal{O}$ и $\vartheta, z \in \mathbb{C}$ равенство (10) остается справедливым при замене $u^{ \pm \kappa}(z)$ на $u_{\vartheta}^{\kappa}(z)$ (это очевидным образом вытекает из (12) при $\kappa \in \mathcal{O} \backslash\{0\}$; в силу леммы 2 можно перейти к пределу $\kappa \rightarrow 0$ и заключить, что то же верно при $\left.\kappa=0^{7)}\right)$.

Определим теперь для каждых $\kappa \in(-1,1)$ и $\vartheta \in \mathbb{R}$ положительную меру Радона $\mathcal{V}_{\kappa, \vartheta}$ на $\mathbb{R}$ следующим образом. Для $0<|\kappa|<1$ положим

$$
\mathcal{V}_{\kappa, \vartheta}= \begin{cases}\widetilde{\mathcal{V}}_{\kappa, \vartheta}, & \vartheta \in\left[-\left|\vartheta_{\kappa}\right|,\left|\vartheta_{\kappa}\right|\right]+\pi \mathbb{Z}, \\ \frac{\pi \sin \pi \kappa\left|E_{\kappa, \vartheta}\right|}{2 \kappa \sin \left(\vartheta+\vartheta_{\kappa}\right) \sin \left(\vartheta-\vartheta_{\kappa}\right)} \delta_{E_{\kappa, \vartheta}}+\widetilde{\mathcal{V}}_{\kappa, \vartheta}, & \vartheta \in\left(\left|\vartheta_{\kappa}\right|, \pi-\left|\vartheta_{\kappa}\right|\right)+\pi \mathbb{Z},\end{cases}
$$

где $\vartheta_{\kappa}$ определено формулой $(14)$, положительная мера Радона $\widetilde{\mathcal{V}}_{\kappa, \vartheta}$ на $\mathbb{R}$ дается соотношением

$$
\begin{aligned}
& d \widetilde{\mathcal{V}}_{\kappa, \vartheta}(E)= \\
& \quad=\frac{1}{2} \frac{\Theta(E) \sin ^{2} \pi \kappa}{E^{-\kappa} \sin ^{2}\left(\vartheta+\vartheta_{\kappa}\right)-2 \cos \pi \kappa \sin \left(\vartheta+\vartheta_{\kappa}\right) \sin \left(\vartheta-\vartheta_{\kappa}\right)+E^{\kappa} \sin ^{2}\left(\vartheta-\vartheta_{\kappa}\right)} d E
\end{aligned}
$$

и $\delta_{E_{\kappa, \vartheta}}$ есть мера Дирака в точке

$$
E_{\kappa, \vartheta}=-\left(\frac{\sin \left(\vartheta+\vartheta_{\kappa}\right)}{\sin \left(\vartheta-\vartheta_{\kappa}\right)}\right)^{1 / \kappa}
$$

При $\kappa=0$ мера $\mathcal{V}_{\kappa, \vartheta}$ определяется при помощи предельного перехода $\kappa \rightarrow 0$ в формулах (16)-(18). Это дает

$$
\mathcal{V}_{0, \vartheta}= \begin{cases}\widetilde{\mathcal{V}}_{0, \vartheta}, & \vartheta \in \pi \mathbb{Z} \\ \frac{\pi^{2}\left|E_{0, \vartheta}\right|}{2 \sin ^{2} \vartheta} \delta_{E_{0, \vartheta}}+\widetilde{\mathcal{V}}_{0, \vartheta}, & \vartheta \notin \pi \mathbb{Z}\end{cases}
$$

6) Чтобы вычислить предел $u_{\vartheta}^{\kappa}(z \mid r)$ при $\kappa \rightarrow 0$, следует применить правило Лопиталя и воспользоваться равенством $\Gamma^{\prime}(1+n) / \Gamma(1+n)=c_{n}-\gamma($ см. [12], раздел 1.7.1, формула $(9))$.

7) Другой способ заключается в том, чтобы выразить $u_{\vartheta}^{0}(z \mid r)$ через функции Бесселя $J_{0}$ и $Y_{0}$ при помощи равенства $\pi Y_{0}(\zeta)=2(\gamma+\ln (\zeta / 2)) J_{0}(\zeta)-2 \mathcal{Y}\left(\zeta^{2}\right)$ (см. [12], раздел 7.2.4, формула (33)) и воспользоваться уравнением Бесселя. 
где

$$
E_{0, \vartheta}=-e^{\pi \operatorname{ctg} \vartheta}
$$

и положительная мера Радона $\widetilde{\mathcal{V}}_{0, \vartheta}$ на $\mathbb{R}$ имеет вид

$$
d \widetilde{\mathcal{V}}_{0, \vartheta}(E)=\frac{1}{2} \frac{\Theta(E)}{\left(\cos \vartheta-\pi^{-1} \ln E \sin \vartheta\right)^{2}+\sin ^{2} \vartheta} d E .
$$

Следующая теорема описывает самосопряженные расширения $h_{\kappa}$ при $-1<\kappa<1$ в терминах их разложений по собственным функциям.

Tеорема 3. Пусть $-1<\kappa<1$. Для любого $\vartheta \in \mathbb{R}$ существует единственный унитарный оператор $U_{\kappa, \vartheta}: L_{2}\left(\mathbb{R}_{+}\right) \rightarrow L_{2}\left(\mathbb{R}, \mathcal{V}_{\kappa, \vartheta}\right)$ такой, что

$$
\left(U_{\kappa, \vartheta} \psi\right)(E)=\int_{0}^{\infty} u_{\vartheta}^{\kappa}(E \mid r) \psi(r) d r, \quad \psi \in L_{2}^{c}\left(\mathbb{R}_{+}\right),
$$

$\partial л я \mathcal{V}_{\kappa, \vartheta}-$-n.в. E. Onepamop

$$
h_{\kappa, \vartheta}=U_{\kappa, \vartheta}^{-1} \mathcal{T}_{\iota}^{\mathcal{V}_{\kappa, \vartheta}} U_{\kappa, \vartheta},
$$

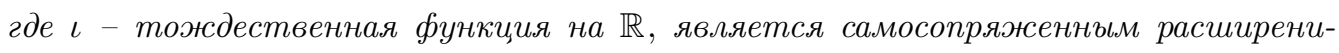
ем $h_{\kappa}$. Обратно, каждое самосопряженное расширение $h_{\kappa}$ равно $h_{\kappa, \vartheta}$ для некоторого $\vartheta \in \mathbb{R}$. Если $\vartheta, \vartheta^{\prime} \in \mathbb{R}$, то равенство $h_{\kappa, \vartheta}=h_{\kappa, \vartheta^{\prime}}$ справедливо тогда и только тогда, когда $\vartheta-\vartheta^{\prime} \in \pi \mathbb{Z}$.

При $\vartheta=\vartheta_{\kappa}$ имеем $\mathcal{V}_{\kappa, \vartheta}=\mathcal{V}_{\kappa}$ и $u^{\kappa}(z)=u_{\vartheta}^{\kappa}(z)$ для всех $z \in \mathbb{C}$, и, следовательно, оператор $U_{\kappa, \vartheta}$ совпадает с преобразованием Ханкеля $U_{\kappa}$.

Разложения, описываемые теоремой 3 , обладают тем преимуществом, что $\kappa=0$ не является точкой разрыва ни для собственных функций $u_{\vartheta}^{\kappa}(E)$, ни для спектральных мер ${ }^{8)} \mathcal{V}_{\kappa, \vartheta}$. Это вытекает из леммы 2 и следующей теоремы.

Теорема 4. Пусть $\varphi$ - непрерывная (ограниченная борелевская) функиия на $\mathbb{R}$ с компактным носителем. Тогда $(\kappa, \vartheta) \rightarrow \int \varphi(E) d \mathcal{V}_{\kappa, \vartheta}(E)$ является непрерывной (борелевской) функиией на $(-1,1) \times \mathbb{R}$, ограниченной на $[-\alpha, \alpha] \times \mathbb{R}$ для любого $0 \leqslant \alpha<1$.

Теоремы 3 и 4 представляют собой главные результаты настоящей работы. Мы также даем новое доказательство теоремы 1 , основанное на локально определенных сингулярных $m$-функциях (см. ниже).

Для доказательства теорем 1 и 3 мы используем вариант теории Титчмарша-Вейля-Кодаиры, недавно развитый в работах [6], [8], где было предложено обобщение понятия $m$-функции Титчмарша-Вейля, применимое не только к задачам с регулярным концом, но и к широкому классу операторов Шредингера с двумя сингулярными концами. Использование таких сингулярных $m$-функций приводит к заметному упрощению при работе с разложениями по собственным функциям по сравнению с общей теорией [13], [5], основанной на матричнозначных мерах (отметим вместе

\footnotetext{
8) В данной статье термин "спектральная мера" всюду используется по отношению к определенным положительным мерам на $\mathbb{R}$, точное определение которых будет дано ниже в предложении 14 . Это отличается от терминологии, принятой в работе [10], где указанный термин применялся к проекторнозначным мерам в гильбертовом пространстве.
} 
с тем, что результаты работ [6], [8] о разложениях по собственным функциям нетрудно вывести из общего подхода Кодаиры [13]; см. замечание 16 ниже).

Статья организована следующим образом. В разделе 2 сообщаются основные факты общей теории самосопряженных расширений одномерных операторов Шредингера и их разложений по собственным функциям. Предложение 14 , являющееся главным утверждением этого раздела, сходно с теоремой 3.4 в [8], но, в отличие от последней, дает локальную версию формулы для спектральных мер, которая позволяет использовать различные $m$-функции для разных областей спектрального параметра. В разделе 3 приводится вывод теоремы 1, иллюстрирующий этот локальный подход к отысканию спектральных мер, и доказывается теорема 3. Раздел 4 посвящен доказательству теоремы 4.

\section{2. ОДНОМЕРНЫЕ ОПЕРАТОРЫ ШРЕДИНГЕРА}

В данном разделе мы напомним основные факты [5], [14], [15], касающиеся самосопряженных расширений одномерных операторов Шредингера, и кратко опишем развитый в работах [6], [8] подход к разложениям по собственным функциям. Отличительной чертой приведенного ниже изложения является использование понятия граничного пространства (см. определение 6 ниже), которое может рассматриваться как формализация концепции самосопряженного граничного условия. Применение граничных пространств во многих случаях дает нам возможность единообразно рассматривать случаи предельной точки и предельной окружности и, тем самым, ведет к более прозрачной формулировке результатов.

Пусть $-\infty \leqslant a<b \leqslant \infty, \lambda_{a, b}$ - сужение на интервал $(a, b)$ меры Лебега $\lambda$ на $\mathbb{R}, \mathcal{D}-$ пространство всех комплексных непрерывно дифференцируемых функций на $(a, b)$, производные которых абсолютно непрерывны на $(a, b)$ (т. е. абсолютно непрерывны на каждом отрезке $[c, d]$, где $a<c \leqslant d<b$ ). Пусть $q-$ комплексная локально интегрируемая функция на $(a, b)$. Для $z \in \mathbb{C}$ обозначим через $l_{q, z}$ такой линейный оператор из $\mathcal{D}$ в пространство комплексных $\lambda_{a, b}$-классов эквивалентности, что

$$
\left(l_{q, z} f\right)(r)=-f^{\prime \prime}(r)+q(r) f(r)-z f(r)
$$

для $\lambda$-п.в. $r \in(a, b)$, и положим

$$
l_{q}=l_{q, 0} .
$$

Для любых $f \in \mathcal{D}$ и $z \in \mathbb{C}$ имеем $l_{q, z} f=l_{q} f-z[f]$, где $[f]=[f]_{\lambda_{a, b}}$ есть $\lambda_{a, b}$-класс эквивалентности функции $f$. Для каждого $c \in(a, b)$ и любых комплексных чисел $z$, $\zeta_{1}$ и $\zeta_{2}$ имеется единственное решение $f$ уравнения $l_{q, z} f=0$ такое, что $f(c)=\zeta_{1}$ и $f^{\prime}(c)=\zeta_{2}$. Отсюда следует, что решения уравнения $l_{q, z} f=0$ образуют двумерное подпространство в $\mathcal{D}$. Для любых функций $f, g \in \mathcal{D}$ их вронскиан $W_{r}(f, g)$ в точке $r \in(a, b)$ определяется соотношением

$$
W_{r}(f, g)=f(r) g^{\prime}(r)-f^{\prime}(r) g(r) \text {. }
$$

Очевидно, что функция $r \rightarrow W_{r}(f, g)$ абсолютно непрерывна на $(a, b)$. Если $f$ и $g$ таковы, что $r \rightarrow W_{r}(f, g)$ есть постоянная функция на $(a, b)$ (в частности, это так, если $f$ и $g$ - решения уравнения $l_{q, z} f=l_{q, z} g=0$ при некотором $z \in \mathbb{C}$ ), то ее 
значение будет обозначаться через $W(f, g)$. Из (23) сразу следует, что для всех $f_{1}, f_{2}, f_{3}, f_{4} \in \mathcal{D}$ и $r \in(a, b)$ верны тождества

$$
\begin{gathered}
W_{r}\left(f_{1}, f_{2}\right) W_{r}\left(f_{3}, f_{4}\right)+W_{r}\left(f_{1}, f_{3}\right) W_{r}\left(f_{4}, f_{2}\right)+W_{r}\left(f_{2}, f_{3}\right) W_{r}\left(f_{1}, f_{4}\right)=0 \\
W_{r}\left(f_{1} f_{2}, f_{3} f_{4}\right)=f_{1}(r) f_{3}(r) W_{r}\left(f_{2}, f_{4}\right)+W_{r}\left(f_{1}, f_{3}\right) f_{2}(r) f_{4}(r) .
\end{gathered}
$$

В оставшейся части данного раздела функция $q$ будет предполагаться вещественной. Пусть

$$
\mathcal{D}_{q}=\left\{f \in \mathcal{D}: f \text { и } l_{q} f \text { квадратично-интегрируемы на }(a, b)\right\} .
$$

Будем говорить, что $\lambda_{a, b}$-измеримая комплексная функция $f$ является квадратично-интегрируемой слева (справа) на $(a, b)$, если $\int_{a}^{c}|f(r)|^{2} d r<\infty\left(\int_{c}^{b}|f(r)|^{2} d x<\infty\right)$ для любого $c \in(a, b)$. Подпространство $\mathcal{D}$, состоящее из таких квадратично-интегрируемых слева (справа) функций $f$ на $(a, b)$, что функция $l_{q} f$ также квадратично-интегрируема слева (справа) на $(a, b)$, будет обозначаться через $\mathcal{D}_{q}^{\mathrm{l}}\left(\mathcal{D}_{q}^{\mathrm{r}}\right)$. Очевидно, имеем $\mathcal{D}_{q}=\mathcal{D}_{q}^{\mathrm{l}} \cap \mathcal{D}_{q}^{\mathrm{r}}$. При помощи интегрирования по частям из (22) следует, что

$$
\int_{c}^{d}\left(\left(l_{q, z} f\right)(r) g(r)-f(r)\left(l_{q, z} g\right)(r)\right) d r=W_{d}(f, g)-W_{c}(f, g)
$$

для любых $f, g \in \mathcal{D}, z \in \mathbb{C}$ и $c, d \in(a, b)$. Поэтому пределы $W_{a}(f, g)=\lim _{r \downarrow a} W_{r}(f, g)$ и $W_{b}(f, g)=\lim _{r \uparrow b} W_{r}(f, g)$ существуют для любых $f, g \in \mathcal{D}_{q}^{\mathrm{l}}$ и $f, g \in \mathcal{D}_{q}^{\mathrm{r}}$ соответственно. Как следствие, имеем

$$
\left\langle l_{q} f,[g]\right\rangle-\left\langle[f], l_{q} g\right\rangle=W_{b}(\bar{f}, g)-W_{a}(\bar{f}, g)
$$

для всех $f, g \in \mathcal{D}_{q}$, где $\langle\cdot, \cdot\rangle$ - скалярное произведение в $L_{2}(a, b)$.

Для любого линейного подпространства $Z$ пространства $\mathcal{D}_{q}$ обозначим через $L_{q}(Z)$ линейный оператор в $L_{2}(a, b)$, определенный соотношениями

$$
\begin{aligned}
D_{L_{q}(Z)} & =\{[f]: f \in Z\}, \\
L_{q}(Z)[f] & =l_{q} f, \quad f \in Z .
\end{aligned}
$$

Определим минимальный оператор $L_{q}$, полагая

$$
L_{q}=L_{q}\left(\mathcal{D}_{q}^{0}\right),
$$

где

$$
\mathcal{D}_{q}^{0}=\left\{f \in \mathcal{D}_{q}: W_{a}(f, g)=W_{b}(f, g)=0 \text { для любого } g \in \mathcal{D}_{q}\right\} .
$$

В силу (26) оператор $L_{q}(Z)$ является симметрическим тогда и только тогда, когда $W_{a}(\bar{f}, g)=W_{b}(\bar{f}, g)$ для всех $f, g \in Z$. В частности, оператор $L_{q}$ симметрический. Кроме того, $L_{q}$ замкнут и плотно определен, а его сопряженный оператор $L_{q}^{*}$ имеет вид

$$
L_{q}^{*}=L_{q}\left(\mathcal{D}_{q}\right)
$$

(см. лемму 9.4 в [14]). Если $T$ - симметрическое расширение $L_{q}$, то $L_{q}^{*}$ - расширение $T^{*}$ и, следовательно, $T$. Ввиду (30) отсюда следует, что $T$ есть оператор вида $L_{q}(Z)$ для некоторого подпространства $Z$ пространства $\mathcal{D}_{q}$. 
ЗАмечАниЕ 5. Самосопряженные операторы вида $L_{q}(Z)$ естественно рассматривать как самосопряженные реализации дифференциального выражения $-d^{2} / d r^{2}+q$. Если оператор $L_{q}(Z)$ самосопряжен, то из равенства (30) и замкнутости $L_{q}$ вытекает, что $L_{q}(Z)$ является расширением $L_{q}$, так как $L_{q}\left(\mathcal{D}_{q}\right)$ есть расширение $L_{q}(Z)$. Таким образом, самосопряженные реализации выражения $-d^{2} / d r^{2}+q-$ это в точности самосопряженные расширения минимального оператора $L_{q}$.

ОПРЕДЕЛЕНИЕ 6. Будем говорить, что линейное подпространство $X$ пространства $\mathcal{D}_{q}^{l}$ является левым граничным пространством, если выполнены следующие условия:

1) $W_{a}(\bar{f}, g)=0$ для всех $f, g \in X$;

2) если $g \in \mathcal{D}_{q}^{1}$ удовлетворяет равенству $W_{a}(\bar{f}, g)=0$ для всех $f \in X$, то $g \in X$. Определение правого граничного пространства получается заменой $\mathcal{D}_{q}^{1}$ на $\mathcal{D}_{q}^{\mathrm{r}}$ и $a$ на $b$.

ОПРЕДЕЛЕНИЕ 7 . Если $W_{a}(f, g)=0$ для всех $f, g \in \mathcal{D}_{q}^{1}$, то говорят, что $q$ отвечает случаю предельной точки (СПТ) в $a$. В противном случае говорят, что $q$ отвечает случаю предельной окружности $(\mathrm{CПО})$ в $a$. Сходным образом $q$ отвечает СПТ в $b$, если $W_{b}(f, g)=0$ для всех $f, g \in \mathcal{D}_{q}^{\mathrm{r}}$ и отвечает СПО в $b$ в противном случае.

Ясно, что $q$ отвечает СПТ в $a($ в $b)$ тогда и только тогда, когда $\mathcal{D}_{q}^{l}-$ единственное левое $\left(\mathcal{D}_{q}^{\mathrm{r}}\right.$ - единственное правое) граничное пространство. Для $f \in \mathcal{D}_{q}^{\mathrm{l}}\left(f \in \mathcal{D}_{q}^{\mathrm{r}}\right)$ положим

$$
\mathcal{D}_{q, f}^{\mathrm{l}}=\left\{g \in \mathcal{D}_{q}^{\mathrm{l}}: W_{a}(\bar{f}, g)=0\right\} \quad\left(\mathcal{D}_{q, f}^{\mathrm{r}}=\left\{g \in \mathcal{D}_{q}^{\mathrm{r}}: W_{b}(\bar{f}, g)=0\right\}\right) .
$$

Для каждого $E \in \mathbb{R}$ обозначим через $S_{q, E}^{\mathrm{l}}\left(S_{q, E}^{\mathrm{r}}\right)$ множество всех таких нетривиальных вещественных элементов $f$ пространства $\mathcal{D}_{q}^{l}\left(\mathcal{D}_{q}^{\mathrm{r}}\right)$, что $l_{q, E} f=0$.

Следующее предложение является переформулировкой известных результатов о самосопряженных расширениях оператора $L_{q}$ (см., например, раздел 9.2 в [14]) на языке граничных пространств.

ПрЕДЛОЖЕНИЕ 8. Пусть $q$ - вещественная локально интегрируемая функиия на $(a, b)$. Тогда верны следующие утверждения.

1. Пусть $X$ и $Y$-соответственно левое и правое граничные пространства. Тогда оператор $L_{q}(X \cap Y)$ является самосопряжсенным расширением $L_{q}$.

2. Предположим, что $L_{q}(X \cap Y)=L_{q}(\tilde{X} \cap \tilde{Y})$ для некоторых левых граничных пространств $X$ и $\widetilde{X}$ и правых граничных пространств $Y$ u $\widetilde{Y}$. Тогда $X=\widetilde{X}$ $u Y=\widetilde{Y}$.

3. Пусть $E \in \mathbb{R} u f \in S_{q, E}^{\mathrm{l}}\left(f \in S_{q, E}^{\mathrm{r}}\right)$. Тогда $\mathcal{D}_{q, f}^{\mathrm{l}}-$ левое $\left(\mathcal{D}_{q, f}^{\mathrm{r}}-\right.$ правое $)$ граничное пространство.

4. Пусть $z \in \mathbb{C}$. Тогда q отвечает СПО в а (в b) в том и только том случае, если каждая функиия $f \in \mathcal{D}$ такая, что $l_{q, z} f=0$ квадратично-интегрируема слева (справа) на $(a, b)$.

5. Если q отвечает СПТ в а или в $b$, то каждое самосопряженное расширение $L_{q}$ совпадает с $L_{q}(X \cap Y)$ для некоторого левого граничного пространства $X$ и правого граничного пространства $Y$.

6. Пусть q отвечает СПО в а (в $b)$ u $E \in \mathbb{R}$. Тогда каждое левое (правое) граничное пространство совпадает с $\mathcal{D}_{q, f}^{\mathrm{l}}\left(\mathcal{D}_{q, f}^{\mathrm{r}}\right)$ для некоторого $f \in S_{q, E}^{\mathrm{l}}\left(f \in S_{q, E}^{\mathrm{r}}\right)$. 
Операторы вида $L_{q}(X \cap Y)$, где $X$ и $Y$ - левое и правое граничные пространства, называются самосопряженными расширениями $L_{q}$ с разделяющимися граничными условиями.

ЗАмЕчАниЕ 9. Как уже упоминалось выше, граничные пространства можно понимать как самосопряженные граничные условия. В этом смысле область определения $L_{q}(X \cap Y)$ состоит из $\left(\lambda_{a, b}\right.$-классов эквивалентности) всех элементов $\mathcal{D}_{q}$, удовлетворяющих слева и справа самосопряженным граничным условиям $X$ и $Y$ соответственно.

ЗАмЕчАниЕ 10. Пусть $f$ и $g$ - линейно независимые решения уравнения $l_{q, z} f=$ $l_{q, z} g=0$, где $\operatorname{Im} z \neq 0$. Предположим, что $f$ удовлетворяет самосопряженному граничному условию в $a$ (т. е. принадлежит некоторому левому граничному пространству). Обозначим через $A$ множество всех таких $\zeta \in \mathbb{C}$, что $g+\zeta f$ принадлежит некоторому правому граничному пространству. Тогда $A$ является либо одноточечным множеством, либо окружностью в зависимости от того, какому случаю в $b$ отвечает $q$ - СПТ или СПО. Более того, $A$ есть предел окружностей $A_{c}$, получающихся при замене $b$ регулярным концом $c \in(a, b)$ в определении множества $A$. Такой предельный переход был исходно использован Вейлем [2] для введения различения между СПТ и СПО.

Если $q$ отвечает СПТ как в $a$, так и в $b$, то из утверждения 1 предложения 8 следует, что оператор $L_{q}\left(\mathcal{D}_{q}\right)$ самосопряжен. Ввиду (30) это означает, что оператор $L_{q}$ самосопряжен.

Для любого $f \in \mathcal{D}_{q}^{1}$ положим

$$
L_{q}^{f}=L_{q}\left(\mathcal{D}_{q, f}^{1} \cap \mathcal{D}_{q}^{\mathrm{r}}\right)
$$

Лемма 11. Пусть $E \in \mathbb{R}$ u q отвечает СПО в а и СПТ в b. Тогда самосопряженные расширения $L_{q}$ - это в точности операторы $L_{q}^{f}$, где $f \in S_{q, E}^{\mathrm{l}}$. Eсли $f, g \in S_{q, E}^{\mathrm{l}}$, то равенство $L_{q}^{f}=L_{q}^{g}$ справедливо тогда и только тогда, когда $g=c f$ для некоторого вещественного $c \neq 0$.

ДокАзАтельство. Первое утверждение сразу следует из утверждений 1, 3, 5 и 6 предложения 8. Пусть $f, g \in S_{q, E}^{\mathrm{l}}$. Если $g=c f$, то в силу (31) имеем

$$
\mathcal{D}_{q, f}^{1}=\mathcal{D}_{q, g}^{1}
$$

и, следовательно, $L_{q}^{f}=L_{q}^{g}$. Обратно, если $L_{q}^{f}=L_{q}^{g}$, то равенство (33) вытекает из утверждений 2 и 3 предложения 8. Поскольку $f \in \mathcal{D}_{q, f}^{1}$ в силу (31), заключаем, что $f \in \mathcal{D}_{q, g}^{1}$. Это означает, что $W_{a}(g, f)=0$. Так как $l_{q, z} f=l_{q, z} g=0$, то $W(g, f)=0$, и потому $g=c f$.

Рассмотрим теперь связанные с $L_{q}$ разложения по собственным функциям.

Пусть $O \subset \mathbb{C}$ - открытое множество. Будем говорить, что отображение $u: O \rightarrow \mathcal{D}$ является $q$-решением в $O$, если $l_{q, z} u(z)=0$ для любого $z \in O$. Назовем $q$-решение $u$ в $O$ аналитическим, если функции $z \rightarrow u(z \mid r)$ и $z \rightarrow \partial_{r} u(z \mid r)$ аналитичны в $O$ для любого $r \in(a, b)$. Назовем $q$-решение $u$ в $O$ необнуляющимся, если $u(z) \neq 0$ для любого $z \in O$, и вещественным, если функция $u(E)$ вещественна для любого $E \in O \cap \mathbb{R}$. 
ОПРЕДЕЛЕНИЕ 12 . Тройка $(q, Y, u)$ называется тройкой разложения, если $q$ - вещественная локально интегрируемая функция на $(a, b), Y$ - правое граничное пространство, $u$ - вещественное необнуляющееся аналитическое $q$-решение в $\mathbb{C}$, удовлетворяющее следующим условиям:

1) $u(z) \in \mathcal{D}_{q}^{l}$ для всех $z \in \mathbb{C}$;

2) найдется такое $E \in \mathbb{R}$, что $W_{a}(u(E), u(z))=0$ для всех $z \in \mathbb{C}$.

Лемма 13. Пусть $\mathfrak{t}=(q, Y, u)-$ тройка разложения. Тогда существует единственное левое граничное пространство $X^{\mathfrak{t}}$ такое, что $u(z) \in X^{\mathfrak{t}}$ для всех $z \in \mathbb{C}$. Для любого $E \in \mathbb{R}$ имеем $X^{\mathfrak{t}}=\mathcal{D}_{q, u(E)}^{1}$.

ДоказАтЕЛьство. Пусть $E \in \mathbb{R}$ и $X$ - левое граничное пространство, содержащее $u(E)$. В силу (31) и условия 1 определения 6 имеем $X \subset \mathcal{D}_{q, u(E)}^{1}$. С другой стороны, если $g \in \mathcal{D}_{q, u(E)}^{1}$, то $W_{a}(\bar{f}, g)=0$ для всех $f \in X$, так как $\mathcal{D}_{q, u(E)}^{1}$ есть левое граничное пространство согласно утверждению 3 предложения 8. Ввиду условия 2 определения 6 заключаем, что $g \in X$ и, следовательно, $X=\mathcal{D}_{q, u(E)}^{1}$. Это означает, что пространство $X^{\mathfrak{t}}$ (если оно существует) единственно и совпадает с $\mathcal{D}_{q, u(E)}^{\mathrm{l}}$ для всех $E \in \mathbb{R}$. В силу (31) и определения 12 найдется такое $E \in \mathbb{R}$, что $u(z) \in \mathcal{D}_{q, u(E)}^{1}$ для всех $z \in \mathbb{C}$. Тем самым доказано существование $X^{\mathfrak{t}}$.

Пусть $\mathfrak{t}=(q, Y, u)$ - тройка разложения, $\tilde{u}$ - такое вещественное аналитическое $q$-решение в $\mathbb{C}$, что $W(u(z), \tilde{u}(z)) \neq 0$ для любого $z \in \mathbb{C}$, и $v$ - такое необнуляющееся аналитическое $q$-решение в $\mathbb{C}_{+}{ }^{9}$ ), что $v(z) \in Y$ для всех $z \in \mathbb{C}_{+}$(такие $\tilde{u}$ и $v$ всегда существуют, см. лемму 2.4 в [8] и лемму 9.8 в [14]). Тогда $W(v(z), u(z)) \neq 0$ для любого $z \in \mathbb{C}_{+}$, поскольку в противном случае мы бы имели $u(z) \in X^{\mathfrak{t}} \cap Y$ и, следовательно, самосопряженный оператор $L_{q}\left(X^{\mathfrak{t}} \cap Y\right)$ обладал бы собственным значением в $\mathbb{C}_{+}$. Определим аналитическую функцию $\mathcal{M}_{\tilde{u}}^{\mathfrak{t}}$ в $\mathbb{C}_{+}$при помощи соотношения

$$
\mathcal{M}_{\tilde{u}}^{\mathfrak{t}}(z)=\frac{1}{\pi} \frac{W(v(z), \tilde{u}(z))}{W(v(z), u(z)) W(u(z), \tilde{u}(z))}
$$

(данное определение, очевидно, не зависит от выбора $v$ ). Следуя [8], будем называть такие функции сингулярными $m$-функциями Титчмарша-Вейля. Мы увидим, что иногда бывает полезно рассматривать $q$-решения $\tilde{u}$, определенные не на всей комплексной плоскости, а на некотором открытом множестве $O \subset \mathbb{C}$. В таком случае будем предполагать, что функция $\mathcal{M}_{\tilde{u}}^{\mathrm{t}}$ определена на $O \cap \mathbb{C}_{+}$.

Обозначим через $L_{2}^{c}(a, b)$ подпространство пространства $L_{2}(a, b)$, состоящее из всех его элементов, обращающихся в нуль $\lambda$-п.в. вне некоторого компактного подмножества $(a, b)$. Следующее предложение дает способ построения разложений по собственным функциям для самосопряженных расширений $L_{q}$ с разделяющимися граничными условиями.

ПреДЛОЖЕНИЕ 14. Пусть $\mathfrak{t}=(q, Y, u)$ - тройка разложения. Тогда верны следующие утверждения.

9) Как обычно, $\mathbb{C}_{+}$обозначает открытую верхнюю полуплоскость комплексной плоскости: $\mathbb{C}_{+}=$ $\{z \in \mathbb{C}: \operatorname{Im} z>0\}$. 
1. Существует единственная положительная мера Радона $\sigma$ на $\mathbb{R}$ (называемая спектральной мерой для t) такая, что

$$
\int \varphi(E) \operatorname{Im} \mathcal{M}_{\tilde{u}}^{\mathfrak{t}}(E+i \eta) d E \rightarrow \int \varphi(E) d \sigma(E) \quad(\eta \downarrow 0)
$$

для любой непрерывной функции $\varphi$ на $\mathbb{R}$ с компактным носителем и любого такого вещественного аналитического q-решения $\tilde{u}$ в $\mathbb{C}$, что $W(u(z), \tilde{u}(z)) \neq 0$ для всех $z \in \mathbb{C}$.

2. Пусть $\sigma$ - спектральная мера для $\mathbf{t}$. Существует единственный унитарный оператор $U: L_{2}(a, b) \rightarrow L_{2}(\mathbb{R}, \sigma)$ (называемый спектралъным преобразованием для $\mathfrak{t}$ ) такой, что

$$
(U \psi)(E)=\int_{a}^{b} u(E \mid r) \psi(r) d r, \quad \psi \in L_{2}^{c}(a, b),
$$

для $\sigma$-n.в. E.

3. Пусть $\sigma$ и $U$ - спектральные мера и преобразование для $\mathfrak{t}$, а левое граничное пространство $X^{\mathfrak{t}}$ удовлетворяет условиям леммы 13. Тогда

$$
L_{q}\left(X^{\mathfrak{t}} \cap Y\right)=U^{-1} \mathcal{T}_{\iota}^{\sigma} U
$$

где ८-тождественная функиия на $\mathbb{R}$.

4. Пусть $\sigma$ - спектральная мера для $\mathfrak{t}, O \subset \mathbb{C}$ - открытое множество, $\tilde{u}$ - такое вещественное аналитическое q-решение в $O$, что $W(u(z), \tilde{u}(z)) \neq 0$ для всех $z \in O$. Тогда

$$
\int_{\operatorname{supp} \varphi} \varphi(E) \operatorname{Im} \mathcal{M}_{\tilde{u}}^{\mathrm{t}}(E+i \eta) d E \rightarrow \int_{O \cap \mathbb{R}} \varphi(E) d \sigma(E) \quad(\eta \downarrow 0)
$$

для любой непрерывной функиии $\varphi$ на $O \cap \mathbb{R}$ с компактным носителем $(\operatorname{supp} \varphi$ обозначает носитель $\varphi$ ).

ДокАзАТЕЛЬство. Утверждения 1-3 представляют собой простую переформулировку соответствующих результатов работы [8] на языке граничных пространств. Пусть $O$ и $\tilde{u}$ удовлетворяют условиям утверждения 4 , а $\theta$ - такое вещественное аналитическое $q$-решение в $\mathbb{C}$, что $W(u(z), \theta(z)) \neq 0$ для всех $z \in \mathbb{C}$. Подстановка $f_{1}=u(z), f_{2}=v(z), f_{3}=\tilde{u}(z)$ и $f_{4}=\theta(z)$ в $(24)$ с последующим делением результата на $\pi W(u(z), v(z)) W(u(z), \theta(z)) W(u(z), \tilde{u}(z))$ приводит к равенству

$$
\mathcal{M}_{\tilde{u}}^{\mathfrak{t}}(z)=\mathcal{M}_{\theta}^{\mathfrak{t}}(z)+\frac{1}{\pi} \frac{W(\tilde{u}(z), \theta(z))}{W(u(z), \theta(z)) W(u(z), \tilde{u}(z))}
$$

для любого $z \in O \cap \mathbb{C}_{+}$. Утверждение 4 теперь следует из утверждения 1 , так как последнее слагаемое в правой части аналитично в $O$ и вещественно на $O \cap \mathbb{R}$.

СлЕДСтвиЕ 15. Пусть $\sigma$ и - спектральные мера и преобразование для тройки разложения $\mathfrak{t}=(q, Y, u)$. Тогда

$$
\left(U^{-1} \varphi\right)(r)=\int u(E \mid r) \varphi(E) d \sigma(E), \quad \varphi \in L_{2}^{c}(\mathbb{R}, \sigma)
$$

для $\lambda$-п.в. $r \in(a, b)$. Если $\sigma(\{E\}) \neq 0$ для некоторого $E \in \mathbb{R}$, то $[u(E)]-$ собственная функиия оператора $L_{q}\left(X^{\mathfrak{t}} \cap Y\right)$. 
ДокАзАТЕЛЬСТво. Для $\varphi \in L_{2}^{c}(\mathbb{R}, \sigma)$ и $r \in(a, b)$ обозначим через $\check{\varphi}(r)$ правую часть (35). Согласно утверждению 2 предложения 14 имеем

$$
\left\langle\psi, U^{-1} \varphi\right\rangle=\langle U \psi, \varphi\rangle=\int \varphi(E) d \sigma(E) \int_{a}^{b} \overline{\psi(r)} u(E \mid r) d r=\int_{a}^{b} \overline{\psi(r)} \check{\varphi}(r) d r
$$

для любого $\psi \in L_{2}^{c}(a, b)$. Отсюда следует равенство (35). В частности, имеем $U^{-1}\left[\chi_{\{E\}}\right]_{\sigma}=\sigma(\{E\})[u(E)]$, где $\chi_{\{E\}}$ - характеристическая функция одноточечного множества $\{E\}$. Согласно утверждению 3 предложения 14 это означает, что $[u(E)]$ является собственной функцией оператора $L_{q}\left(X^{\mathfrak{t}} \cap Y\right)$ при условии $\sigma(\{E\}) \neq 0$.

ЗАмЕчАниЕ 16. Приведенное выше доказательство предложения 14 опирается на результаты работы [8]. В то же время это утверждение нетрудно вывести исходя из общего подхода Кодаиры [13], основанного на использовании матричнозначных мер. В самом деле, если положить $s_{1}(z)=\tilde{u}(z) / W(u(z), \tilde{u}(z))$ и $s_{2}(z)=u(z)$ для $z \in \mathbb{C}$, то единственный невещественный элемент $M_{22}(z)$ характеристической матрицы $M$, определенной формулой (1.13) в работе [13], будет равен $\pi \mathcal{M}_{\tilde{u}}^{\mathfrak{t}}(z)$, и, следовательно, в этом случае теорема 1.3 в [13] в основном совпадает с утверждениями 1-3 предложения 14. Данное в [8] простое прямое доказательство использует лишь одну $m$-функцию и обходится без привлечения матричнозначных мер. Оно существенным образом опирается на технику, развитую в статье [6] для потенциалов, отвечающих СПТ на обоих концах рассматриваемого интервала (анализ в том же духе для СПО на одном из концов можно найти в [16]). Подобный подход к отысканию спектральных мер был также предложен в работе [9] в контексте уравнения Шредингера с обратноквадратичным потенциалом.

Если функция $q$ локальноинтегрируема на $(a, b)$, то из формул $(28)$ и (29) следует, что пространство $C_{0}^{\infty}(a, b)$ гладких функций на $(a, b)$ с компактным носителем содержится в $\mathcal{D}_{q}^{0}$, а $L_{q}$ является расширением $L_{q}\left(C_{0}^{\infty}(a, b)\right)$. Доказательство следующей леммы приведено в приложении Б.

ЛЕмма 17. Пусть $q$ - вещественная локально интегрируемая функиия на $(a, b)$. Тогда $L_{q}$ есть замыкание $L_{q}\left(C_{0}^{\infty}(a, b)\right)$.

\section{3. РАЗЛОЖЕНИЯ ПО СОБСТВЕННЫМ ФУНКЦИЯМ ДЛЯ ОБРАТНОКВАДРАТИЧНОГО ПОТЕНЦИАЛА}

Предположим теперь, что $a=0$ и $b=\infty$, и применим приведенную выше общую теорию к потенциалу $q_{\kappa}$, определенному формулой (5). Из (4) и (27) сразу следует, что $\breve{h}_{\kappa}=L_{q_{\kappa}}\left(C_{0}^{\infty}\left(\mathbb{R}_{+}\right)\right)$. Ввиду (6) и леммы 17 отсюда вытекает, что

$$
h_{\kappa}=L_{q_{\kappa}}, \quad \kappa \in \mathbb{R} .
$$

Уравнение $l_{q_{\kappa}} f=0$ имеет линейно независимые решения $r^{1 / 2 \pm \kappa}$ при $\kappa \neq 0$ и решения $r^{1 / 2}$ и $r^{1 / 2} \ln r$ при $\kappa=0$. В силу утверждения 4 предложения 8 можно сделать следующие заключения:

1) $q_{\kappa}$ отвечает СПТ в точках 0 и $\infty$ для таких вещественных $\kappa$, что $|\kappa| \geqslant 1$;

2) $q_{\kappa}$ отвечает СПТ в $\infty$ и СПО в 0 при $-1<\kappa<1$.

Поэтому оператор $h_{\kappa}$ самосопряжен при $|\kappa| \geqslant 1$ и имеет различные самосопряженные расширения при $-1<\kappa<1$. 
Для любого $\kappa \in \mathbb{C}$ определим отображение $u^{\kappa}: \mathbb{C} \rightarrow \mathcal{D}$ при помощи (7). В силу (10) имеем

$$
l_{q_{\kappa}, z} u^{ \pm \kappa}(z)=0, \quad z, \kappa \in \mathbb{C} .
$$

Мы будем в дальнейшем систематически пользоваться обозначением (15) для комплексной плоскости с разрезом вдоль луча. Обозначим через $\ln$ ветвь логарифма в $\mathbb{C}_{3 \pi / 2}$, удовлетворяющую условию $\ln 1=0$, и положим $z^{\rho}=e^{\rho \ln z}$ для всех $z \in \mathbb{C}_{3 \pi / 2}$ и $\rho \in \mathbb{C}$.

Для каждого $\kappa \in \mathbb{C}$ определим отображение $v^{\kappa}: \mathbb{C}_{3 \pi / 2} \rightarrow \mathcal{D}$ при помощи соотношения

$$
v^{\kappa}(z \mid r)=\frac{i \pi}{2} e^{i \pi \kappa / 2} r^{1 / 2} H_{\kappa}^{(1)}\left(r z^{1 / 2}\right), \quad r \in \mathbb{R}_{+}, \quad z \in \mathbb{C}_{3 \pi / 2},
$$

где $H_{\kappa}^{(1)}$ - функция Ханкеля первого рода порядка $\kappa$. Поскольку $H_{\kappa}^{(1)}$ - решение уравнения Бесселя, имеем

$$
l_{q_{\kappa}, z} v^{\kappa}(z)=0
$$

для любых $z \in \mathbb{C}_{3 \pi / 2}$ и $\kappa \in \mathbb{C}$. Из равенства $H_{-\kappa}^{(1)}=e^{i \pi \kappa} H_{\kappa}^{(1)}$ ([12], раздел 7.2.1, формула (9)) вытекает, что

$$
v^{-\kappa}(z)=v^{\kappa}(z), \quad \kappa \in \mathbb{C}, \quad z \in \mathbb{C}_{3 \pi / 2} .
$$

Пользуясь известным асимптотическим видом функции $H_{\kappa}^{(1)}(\zeta)$ при $\zeta \rightarrow \infty$ (см. [12], раздел 7.13.1, формула (1)), находим, что

$$
v^{\kappa}(z \mid r) \sim 2^{-1} \sqrt{\pi}(i+1) z^{-1 / 4} e^{i z^{1 / 2} r}, \quad r \rightarrow \infty,
$$

для любых $\kappa \in \mathbb{C}$ и $z \in \mathbb{C}_{3 \pi / 2}$, и, следовательно, функция $v^{\kappa}(z)$ квадратично-интегрируема справа для всех $\kappa \in \mathbb{C}$ и $z \in \mathbb{C}_{+}$. С учетом выражения

$$
W_{z}\left(J_{\kappa}, H_{\kappa}^{(1)}\right)=\frac{2 i}{\pi z}
$$

для вронскиана функций Бесселя ([12], раздел 7.11, формула (29)) и равенства (40) из формул (7), (9) и (38) следует, что

$$
W\left(v^{\kappa}(z), u^{\kappa}(z)\right)=z^{-\kappa / 2} e^{i \pi \kappa / 2}, \quad W\left(v^{\kappa}(z), u^{-\kappa}(z)\right)=z^{\kappa / 2} e^{-i \pi \kappa / 2}
$$

для любых $\kappa \in \mathbb{C}$ и $z \in \mathbb{C}_{3 \pi / 2}$.

ЛЕмма 18. Пусть $\kappa>-1$. Тогда $u^{\kappa}(z)$ является нетривиальным элементом $\mathcal{D}_{q_{\kappa}}^{1}$ для любого $z \in \mathbb{C} u W_{0}\left(u^{\kappa}(z), u^{\kappa}\left(z^{\prime}\right)\right)=0$ для всех $z, z^{\prime} \in \mathbb{C}$.

ДокАЗАТЕЛьство. Поскольку $\kappa>-1$, из $(7)$ следует, что функция $u^{\kappa}(z)$ квадратично-интегрируема слева для всех $z \in \mathbb{C}$. Согласно $(37)$ это означает, что $u^{\kappa}(z) \in \mathcal{D}_{q_{\kappa}}^{1}$ для всех $z \in \mathbb{C}$. При $z \neq 0$ функция $u^{\kappa}(z)$ нетривиальна в силу $(7)$, так как в противном случае функция $\mathcal{X}_{\kappa}$ была бы тождественно равна нулю. Поскольку $u^{\kappa}(0 \mid r)=2^{-\kappa} r^{1 / 2+\kappa} / \Gamma(\kappa+1)$ в силу (7) и (8), заключаем, что функция $u^{\kappa}(0)$ нетривиальна при $\kappa>-1$. Согласно (7) и (25) имеем

$$
W_{r}\left(u^{\kappa}(z), u^{\kappa}\left(z^{\prime}\right)\right)=2 r^{2+2 \kappa}\left(z^{\prime} \mathcal{X}_{\kappa}\left(r^{2} z\right) \mathcal{X}_{\kappa}^{\prime}\left(r^{2} z^{\prime}\right)-z \mathcal{X}_{\kappa}^{\prime}\left(r^{2} z\right) \mathcal{X}_{\kappa}\left(r^{2} z^{\prime}\right)\right)
$$

и, следовательно, $W_{0}\left(u^{\kappa}(z), u^{\kappa}\left(z^{\prime}\right)\right)=0$ для всех $z, z^{\prime} \in \mathbb{C}$. 
В силу $(37) u^{\kappa}$ является вещественным аналитическим $q_{\kappa}$-решением в $\mathbb{C}$ для любого $\kappa \in \mathbb{R}$. Так как $q_{\kappa}$ отвечает СПТ в $\infty, \mathcal{D}_{q_{\kappa}}^{\mathrm{r}}$ есть правое граничное пространство для всех $\kappa \in \mathbb{R}$. Поэтому из определения 12 и леммы 18 вытекает, что $\mathfrak{t}_{\kappa}=\left(q_{\kappa}, \mathcal{D}_{q_{\kappa}}^{\mathrm{r}}, u^{\kappa}\right)$ является тройкой разложения для любого $\kappa>-1$. Обозначим через $\sigma_{\kappa}$ спектральную меру для $\mathfrak{t}_{\kappa}$.

Лемма 19. Пусть $\kappa>-1$. Тогда $\sigma_{\kappa}=\mathcal{V}_{\kappa}$, где $\mathcal{V}_{\kappa}-$ мера на $\mathbb{R}$, определяемая формулой (11).

ДокАзАтЕльство. В силу (38), (39) и (41) $v^{\kappa}$ есть необнуляющееся аналитическое $q_{\kappa}$-решение в $\mathbb{C}_{3 \pi / 2}$, удовлетворяющее условию $v^{\kappa}(z) \in \mathcal{D}_{q_{\kappa}}^{\mathrm{r}}$ для любого $z \in \mathbb{C}_{+}$. Обозначим через $\tilde{u}_{1}$ сужение $\left.v^{\kappa}\right|_{O}$ отображения $v^{\kappa}$ на область $O=\{z \in \mathbb{C}: \operatorname{Re} z<0\}$. Согласно (38) при $E<0$ имеем $\tilde{u}_{1}(E)=r^{1 / 2} K_{\kappa}(r \sqrt{|E|})$, где $K_{\kappa}$ - модифицированная функция Бесселя второго рода порядка $\kappa$ ([12], раздел 7.2.2, формула (15)). Поэтому функция $\tilde{u}_{1}(E)$ вещественна для вещественных $E<0$. В силу (43) для всех $z \in O$ имеем $W\left(\tilde{u}_{1}(z), u^{\kappa}(z)\right) \neq 0$. Подставляя $\mathfrak{t}=\mathfrak{t}_{\kappa}, v=\left.v^{\kappa}\right|_{\mathbb{C}_{+}}$и $\tilde{u}=\tilde{u}_{1}$ в $(34)$, находим, что $\mathcal{M}_{\tilde{u}_{1}}^{\mathfrak{t}_{\kappa}}(z)=0$ для всех $z \in O \cap \mathbb{C}_{+}$. В силу утверждения 4 предложения 14 заключаем, что $\sigma_{\kappa}$ обращается в нуль и, как следствие, совпадает с $\mathcal{V}_{\kappa}$ на $(-\infty, 0)$. Зададим отображение $\tilde{u}_{2}: \mathbb{C}_{\pi} \rightarrow \mathcal{D}$ равенством $\tilde{u}_{2}(z \mid r)=r^{1 / 2} Y_{\kappa}\left(r z^{1 / 2}\right)$, где $Y_{\kappa}$ - функция Бесселя второго рода порядка $\kappa$. Так как $Y_{\kappa}$ удовлетворяет уравнению Бесселя, то для любого $z \in \mathbb{C}_{\pi}$ имеем $l_{q_{\kappa}, z} \tilde{u}_{2}(z)=0$, и, следовательно, $\tilde{u}_{2}$ является аналитическим $q_{\kappa}$-решением в $\mathbb{C}_{\pi}$. Поскольку функция $Y_{\kappa}$ вещественна для положительных вещественных значений аргумента, функция $\tilde{u}_{2}(E)$ вещественна при $E>0$. Так как $H_{\kappa}^{(1)}=J_{\kappa}+i Y_{\kappa}$, то из (42) следует, что

$$
W_{z}\left(J_{\kappa}, Y_{\kappa}\right)=W_{z}\left(H_{\kappa}^{(1)}, Y_{\kappa}\right)=-i W_{z}\left(J_{\kappa}, H_{\kappa}^{(1)}\right)=\frac{2}{\pi z} .
$$

В силу (7), (9) и (38) находим, что $W\left(u^{\kappa}(z), \tilde{u}_{2}(z)\right)=2 z^{-\kappa / 2} / \pi \neq 0$ при $z \in \mathbb{C}_{\pi}$ и $W\left(v^{\kappa}(z), \tilde{u}_{2}(z)\right)=i e^{i \pi \kappa / 2}$ при $z \in \mathbb{C}_{+}$. Ввиду (43) подстановка $\mathfrak{t}=\mathfrak{t}_{\kappa}, v=\left.v^{\kappa}\right|_{\mathbb{C}_{+}}$ и $\tilde{u}=\tilde{u}_{2}$ в (34) дает равенство

$$
\mathcal{M}_{\tilde{u}_{2}}^{\mathfrak{t}_{\kappa}}(z)=\frac{i z^{\kappa}}{2}, \quad z \in \mathbb{C}_{+} .
$$

Поэтому согласно утверждению 4 предложения 14 заключаем, что $\sigma_{\kappa}$ совпадает с $\mathcal{V}_{\kappa}$ на $(0, \infty)$. Остается заметить, что $\sigma_{\kappa}(\{0\})=0$, потому что в противном случае функция $u^{\kappa}(0)$ была бы квадратично-интегрируемой в силу следствия 15.

ДОКАЗАТЕЛЬСТВо тЕОРЕМЫ 1. Из утверждения 2 предложения 14 и леммы 19 следует, что оператор $U_{\kappa}$ существует и совпадает со спектральным преобразованием для $\mathfrak{t}_{\kappa}$. Поэтому в силу утверждения 1 предложения 8, утверждения 3 предложения 14, формулы (36) и леммы 19 заключаем, что $U_{\kappa}^{-1} \mathcal{T}_{\iota} \mathcal{V}_{\kappa} U_{\kappa}$ представляет собой самосопряженное расширение $h_{\kappa}$. При $\kappa \geqslant 1$ оператор $h_{\kappa}$ самосопряжен и, следовательно, совпадает со своим самосопряженным расширением $U_{\kappa}^{-1} \mathcal{T}_{\iota} \mathcal{V}_{\kappa} U_{\kappa}$.

ЗАмечАние 20. Как уже отмечалось в разделе 1 , оператор $U_{\kappa}$ в основном совпадает с преобразованием Ханкеля. В работах [6], [8], где данное преобразование рассматривалось сходным образом, требовалось, чтобы второе решение $\tilde{u}$, используемое при вычислении спектральной меры, было глобально определено. Это приводило к необходимости различать целые и нецелые значения $\kappa$. Использование 
локально определенного отображения $\tilde{u}$ в доказательстве леммы 19 позволило нам единообразно рассматривать все значения $\kappa$.

Для $\kappa \in \mathcal{O}$ и $\vartheta \in \mathbb{C}$ определим отображение $u_{\vartheta}^{\kappa}: \mathbb{C} \rightarrow \mathcal{D}$ формулами (12) и (13). Так как равенство (10) остается верным при замене $u^{ \pm \kappa}(z)$ на $u_{\vartheta}^{\kappa}(z)$ (см. раздел 1$)$, то

$$
l_{q_{\kappa}, z} u_{\vartheta}^{\kappa}(z)=0, \quad \kappa \in \mathcal{O}, \quad \vartheta, z \in \mathbb{C} .
$$

В силу (12) и (43) имеем

$$
W\left(v^{\kappa}(z), u_{\vartheta}^{\kappa}(z)\right)=\frac{z^{-\kappa / 2} e^{i \pi \kappa / 2}}{\sin \pi \kappa}\left(\sin \left(\vartheta+\vartheta_{\kappa}\right)-e^{-i \pi \kappa} z^{\kappa} \sin \left(\vartheta-\vartheta_{\kappa}\right)\right)
$$

для всех $\kappa \in \mathcal{O} \backslash\{0\}, \vartheta \in \mathbb{C}$ и $z \in \mathbb{C}_{3 \pi / 2}$. Согласно лемме 2 функция $\kappa \rightarrow$ $W\left(v^{\kappa}(z), u_{\vartheta}^{\kappa}(z)\right)$ аналитична в $\mathcal{O}$ при фиксированных $\vartheta$ и $z$, и, следовательно, величину $W\left(v^{0}(z), u_{\vartheta}^{0}(z)\right)$ можно найти, вычисляя предел $\kappa \rightarrow 0$ в (45). В результате получаем

$$
W\left(v^{0}(z), u_{\vartheta}^{0}(z)\right)=\cos \vartheta+\left(i-\pi^{-1} \ln z\right) \sin \vartheta, \quad \vartheta \in \mathbb{C}, \quad z \in \mathbb{C}_{3 \pi / 2} .
$$

Для любых $\kappa \in \mathcal{O}$ и $z \in \mathbb{C}$ положим

$$
w^{\kappa}(z)=u_{\pi / 2+\vartheta_{\kappa}}^{\kappa}(z),
$$

где $\vartheta_{\kappa}$ задается формулой (14). Из (12) и (13) следует, что

$$
\begin{aligned}
w^{\kappa}(z) & =\frac{u^{\kappa}(z) \cos \pi \kappa-u^{-\kappa}(z)}{\sin \pi \kappa}, \quad \kappa \in \mathcal{O} \backslash\{0\}, \\
w^{0}(z \mid r) & =\frac{2}{\pi}\left[\left(\ln \frac{r}{2}+\gamma\right) u^{0}(z \mid r)-\sqrt{r} \mathcal{Y}\left(r^{2} z\right)\right], \quad r \in \mathbb{R}_{+},
\end{aligned}
$$

для любого $z \in \mathbb{C}$ и

$$
u_{\vartheta}^{\kappa}(z)=u^{\kappa}(z) \cos \left(\vartheta-\vartheta_{\kappa}\right)+w^{\kappa}(z) \sin \left(\vartheta-\vartheta_{\kappa}\right)
$$

для всех $\kappa \in \mathcal{O}$ и $\vartheta, z \in \mathbb{C}$. В силу (44) и (47) имеем

$$
l_{q_{\kappa}, z} w^{\kappa}(z)=0, \quad \kappa \in \mathcal{O}, \quad z \in \mathbb{C} .
$$

Лемма 21. Пусть $-1<\kappa<1$. Тогда $u^{\kappa}(z), w^{\kappa}(z) \in \mathcal{D}_{q_{\kappa}}^{1}$ для любого $z \in \mathbb{C} u$

$$
W_{0}\left(w^{\kappa}(z), w^{\kappa}\left(z^{\prime}\right)\right)=0, \quad W_{0}\left(u^{\kappa}(z), w^{\kappa}\left(z^{\prime}\right)\right)=\frac{2}{\pi}
$$

для всех $z, z^{\prime} \in \mathbb{C}$.

ДоказАтеЛьство. Поскольку $q_{\kappa}$ отвечает СПО в 0 при $-1<\kappa<1$, из утверждения 4 предложения 8 и равенств $(37)$ и $(51)$ вытекает, что $u^{\kappa}(z), w^{\kappa}(z) \in \mathcal{D}_{q_{\kappa}}^{1}$ для любого $z \in \mathbb{C}$. Для $z \in \mathbb{C}$ и $-1<\kappa<1$ определим гладкую функцию $a_{z}^{\kappa}$ на $\mathbb{R}$, полагая $a_{z}^{\kappa}(r)=\mathcal{X}_{\kappa}\left(r^{2} z\right)$, где $\mathcal{X}_{\kappa}$ задается формулой (8). Для $r \in \mathbb{R}_{+}$имеем $u^{\kappa}(z \mid r)=r^{1 / 2+\kappa} a_{z}^{\kappa}(r)$. Поэтому из $(25)$ получаем

$$
W_{r}\left(u^{\kappa}(z), u^{-\kappa}\left(z^{\prime}\right)\right)=r W_{r}\left(a_{z}^{\kappa}, a_{z^{\prime}}^{-\kappa}\right)-2 \kappa a_{z}^{\kappa}(r) a_{z^{\prime}}^{-\kappa}(r)
$$


для любых $r \in \mathbb{R}_{+}$и $z, z^{\prime} \in \mathbb{C}$. Поскольку $a_{z}^{\kappa}(0)=2^{-\kappa} / \Gamma(1+\kappa)$ для любого $z \in \mathbb{C}$, заключаем, что $W_{0}\left(u^{\kappa}(z), u^{-\kappa}\left(z^{\prime}\right)\right)=-2 \sin \pi \kappa / \pi$. Утверждение леммы для $0<|\kappa|<1$ теперь следует из (48) и леммы 18 . Для $z \in \mathbb{C}$ определим гладкую функцию $b_{z}$ на $\mathbb{R}$, полагая

$$
b_{z}(r)=(\gamma-\ln 2) \mathcal{X}_{0}\left(r^{2} z\right)-\mathcal{Y}\left(r^{2} z\right)
$$

В силу (49) имеем

$$
\frac{1}{2} \pi w^{0}(z \mid r)=r^{1 / 2} \ln r a_{z}^{0}(r)+r^{1 / 2} b_{z}(r)
$$

для любого $r \in \mathbb{R}_{+}$. Поэтому из (25) находим

$$
\begin{aligned}
\frac{\pi}{2} W_{r}\left(u^{0}(z), w^{0}\left(z^{\prime}\right)\right)= & r W_{r}\left(a_{z}^{0}, b_{z^{\prime}}\right)+r \ln r W_{r}\left(a_{z}^{0}, a_{z^{\prime}}^{0}\right)+a_{z}^{0}(r) a_{z^{\prime}}^{0}(r), \\
\frac{\pi^{2}}{4} W_{r}\left(w^{0}(z), w^{0}\left(z^{\prime}\right)\right)= & r \ln ^{2} r W_{r}\left(a_{z}^{0}, a_{z^{\prime}}^{0}\right)+r \ln r\left(W_{r}\left(a_{z}^{0}, b_{z^{\prime}}\right)+W_{r}\left(b_{z}, a_{z^{\prime}}^{0}\right)\right)+ \\
& +r W_{r}\left(b_{z}, b_{z^{\prime}}\right)+b_{z}(r) a_{z^{\prime}}^{0}(r)-a_{z}^{0}(r) b_{z^{\prime}}(r)
\end{aligned}
$$

для любых $r \in \mathbb{R}_{+}$и $z, z^{\prime} \in \mathbb{C}$. Поскольку $a_{z}^{0}(0)=1$ и $b_{z}(0)=\gamma-\ln 2$ для любого $z \in \mathbb{C}$, отсюда следует требуемое утверждение при $\kappa=0$.

С учетом (37) и (51) из леммы 21 вытекает, что

$$
W\left(u^{\kappa}(z), w^{\kappa}(z)\right)=\frac{2}{\pi}
$$

для любых $z \in \mathbb{C}$ и $-1<\kappa<1$ (и, следовательно, для всех $z \in \mathbb{C}$ и $\kappa \in \mathcal{O}$ ), и в силу (50) получаем

$$
W\left(u_{\vartheta}^{\kappa}(z), u_{\vartheta-\pi / 2}^{\kappa}(z)\right)=-\frac{2}{\pi}, \quad \vartheta, z \in \mathbb{C}, \quad \kappa \in \mathcal{O} .
$$

Пусть $-1<\kappa<1$ и $\vartheta \in \mathbb{R}$. Согласно (44) и (53) отображение $u_{\vartheta}^{\kappa}$ является вещественным необнуляющимся аналитическим $q_{\kappa}$-решением в $\mathbb{C}$. В силу $(50)$, леммы 18 и леммы 21 имеем $u_{\vartheta}^{\kappa}(z) \in \mathcal{D}_{q_{\kappa}}^{1}$ для всех $z \in \mathbb{C}$ и $W_{0}\left(u_{\vartheta}^{\kappa}(z), u_{\vartheta}^{\kappa}\left(z^{\prime}\right)\right)=0$ для всех $z, z^{\prime} \in \mathbb{C}$. Так как $\mathcal{D}_{q_{\kappa}}^{\mathrm{r}}-$ правое граничное пространство, то из определения 12 следует, что $\mathfrak{t}_{\kappa, \vartheta}=\left(q_{\kappa}, \mathcal{D}_{q_{\kappa}}^{\mathrm{r}}, u_{\vartheta}^{\kappa}\right)$ есть тройка разложения. Обозначим через $\sigma_{\kappa, \vartheta}$ спектральную меру для $\mathfrak{t}_{\kappa, \vartheta}$.

Лемма 22. Пусть $-1<\kappa<1 u \vartheta \in \mathbb{R}$. Тогда $\sigma_{\kappa, \vartheta}=\mathcal{V}_{\kappa, \vartheta}$, где $\mathcal{V}_{\kappa, \vartheta}-$ мера на $\mathbb{R}$, задаваемая формулами (16)-(21).

ДокАЗАтЕльство. В силу (38), (39) и (41) отображение $v^{\kappa}$ является таким необ-

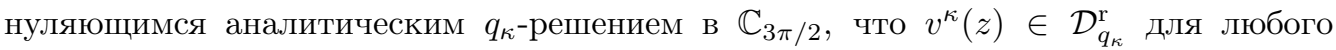
$z \in \mathbb{C}_{+}$. Определим мероморфную функцию $\mathscr{M}_{\kappa, \vartheta}$ в $\mathbb{C}_{3 \pi / 2}$ соотношением

$$
\mathscr{M}_{\kappa, \vartheta}(z)=-\frac{1}{2} \frac{W\left(v^{\kappa}(z), u_{\vartheta-\pi / 2}^{\kappa}(z)\right)}{W\left(v^{\kappa}(z), u_{\vartheta}^{\kappa}(z)\right)}, \quad z \in \mathbb{C}_{3 \pi / 2} .
$$

Подставляя $\mathfrak{t}=\mathfrak{t}_{\kappa, \vartheta}$ и $v=\left.v^{\kappa}\right|_{\mathbb{C}_{+}}$в $(34)$ и учитывая $(53)$, заключаем, что $\mathscr{M}_{\kappa, \vartheta}$ совпадает на $\mathbb{C}_{+}$с сингулярной $m$-функцией Титчмарша-Вейля $\mathcal{M}_{\tilde{u}}^{\mathfrak{t}_{\kappa, \vartheta}}$ для $\tilde{u}=u_{\vartheta-\pi / 2}^{\kappa}$. Согласно утверждению 1 предложения 14 имеем

$$
\int \varphi(E) d \sigma_{\kappa, \vartheta}(E)=\lim _{\eta \downarrow 0} \int \varphi(E) \operatorname{Im} \mathscr{M}_{\kappa, \vartheta}(E+i \eta) d E
$$


для любой непрерывной функции $\varphi$ на $\mathbb{R}$ с компактным носителем. Заметим, что $\sigma_{\kappa, \vartheta}(\{0\})=0$, потому что в противном случае функция $u_{\vartheta}^{\kappa}(0)$ была бы квадратично-интегрируемой в силу следствия 15. Поэтому достаточно показать, что $\sigma_{\kappa, \vartheta}$ совпадает с $\mathcal{V}_{\kappa, \vartheta}$ на интервалах $(-\infty, 0)$ и $(0, \infty)$. Это нетрудно сделать, пользуясь представлением $(55)$ для $\sigma_{\kappa, \vartheta}$. Поскольку явные выражения для $\mathscr{M}_{\kappa, \vartheta}$ отличаются друг от друга при $0<|\kappa|<1$ и $\kappa=0$, мы рассмотрим эти случаи по отдельности.

1. Случай $0<|\kappa|<1$. Согласно (45) и (54) имеем

$$
\mathscr{M}_{\kappa, \vartheta}(z)=\frac{1}{2} \frac{\cos \left(\vartheta+\vartheta_{\kappa}\right)-e^{-i \pi \kappa} z^{\kappa} \cos \left(\vartheta-\vartheta_{\kappa}\right)}{\sin \left(\vartheta+\vartheta_{\kappa}\right)-e^{-i \pi \kappa} z^{\kappa} \sin \left(\vartheta-\vartheta_{\kappa}\right)}
$$

Легко видеть, что $\mathscr{M}_{\kappa, \vartheta}$ не имеет особенностей на $(0, \infty)$ и

$$
\operatorname{Im} \mathscr{M}_{\kappa, \vartheta}(E)=\frac{1}{2} \frac{\sin ^{2} \pi \kappa}{E^{-\kappa} \sin ^{2} \vartheta_{+}-2 \cos \pi \kappa \sin \vartheta_{+} \sin \vartheta_{-}+E^{\kappa} \sin ^{2} \vartheta_{-}}, \quad E>0,
$$

где $\vartheta_{ \pm}=\vartheta \pm \vartheta_{\kappa}$. В силу (16), (17) и (55) заключаем, что $\sigma_{\kappa, \vartheta}$ совпадает с $\mathcal{V}_{\kappa, \vartheta}$ на $(0, \infty)$. При $\vartheta \in\left[-\left|\vartheta_{\kappa}\right|,\left|\vartheta_{\kappa}\right|\right]+\pi \mathbb{Z}$ функция $\mathscr{M}_{\kappa, \vartheta}$ вещественна на $(-\infty, 0)$ и не имеет особенностей на этом множестве. Поэтому из формулы (55) следует, что $\sigma_{\kappa, \vartheta}$ обращается в нуль на $(-\infty, 0)$ для таких $\vartheta$. Если $\vartheta \in\left(\left|\vartheta_{\kappa}\right|, \pi-\left|\vartheta_{\kappa}\right|\right)+\pi \mathbb{Z}$, то функция $\mathscr{M}_{\kappa, \theta}$ имеет простой полюс в точке $E_{\kappa, \vartheta}$, определенной формулой (18), и, следовательно, представима в виде

$$
\mathscr{M}_{\kappa, \vartheta}(z)=g(z)+\frac{A}{E_{\kappa, \vartheta}-z},
$$

где $g$ - функция, аналитическая в $\mathbb{C}_{3 \pi / 2}$ и вещественная на $(-\infty, 0)$, и

$$
A=\lim _{z \rightarrow E_{\kappa, \vartheta}}\left(E_{\kappa, \vartheta}-z\right) \mathscr{M}_{\kappa, \vartheta}(z)=\frac{\sin \pi \kappa\left|E_{\kappa, \vartheta}\right|}{2 \kappa \sin \left(\vartheta+\vartheta_{\kappa}\right) \sin \left(\vartheta-\vartheta_{\kappa}\right)} .
$$

Поэтому из (55) вытекает, что мера $\sigma_{\kappa, \vartheta}$ равна $\pi A \delta_{E_{\kappa, \vartheta}}$ на $(-\infty, 0)$. Таким образом, $\sigma_{\kappa, \vartheta}$ совпадает с $\mathcal{V}_{\kappa, \vartheta}$ на $(-\infty, 0)$ для всех $\vartheta$.

2. Случай $\kappa=0$. Согласно (46) и (54) имеем

$$
\mathscr{M}_{0, \vartheta}(z)=\frac{1}{2} \frac{\left(i-\pi^{-1} \ln z\right) \cos \vartheta-\sin \vartheta}{\cos \vartheta+\left(i-\pi^{-1} \ln z\right) \sin \vartheta} .
$$

Легко видеть, что $\mathscr{M}_{0, \vartheta}$ не имеет особенностей на $(0, \infty)$ и

$$
\operatorname{Im} \mathscr{M}_{0, \vartheta}(E)=\frac{1}{2} \frac{1}{\left(\cos \vartheta-\pi^{-1} \ln E \sin \vartheta\right)^{2}+\sin ^{2} \vartheta}, \quad E>0 .
$$

В силу $(19),(21)$ и $(55)$ заключаем, что $\sigma_{0, \vartheta}$ совпадает с $\mathcal{V}_{0, \vartheta}$ на $(0, \infty)$. При $\vartheta \in \pi \mathbb{Z}$ функция $\mathscr{M}_{0, \vartheta}$ вещественна на $(-\infty, 0)$ и не имеет особенностей на этом множестве. Поэтому из формулы (55) следует, что мера $\sigma_{0, \vartheta}$ обращается в нуль на $(-\infty, 0)$ для таких $\vartheta$. Если $\vartheta \notin \pi \mathbb{Z}$, то функция $\mathscr{M}_{0, \theta}$ имеет простой полюс в точке $E_{0, \vartheta}$, определенной формулой (20), и, следовательно, представима в виде

$$
\mathscr{M}_{0, \vartheta}(z)=g(z)+\frac{A}{E_{0, \vartheta}-z},
$$


где $g$ - функция, аналитическая в $\mathbb{C}_{3 \pi / 2}$ и вещественная на $(-\infty, 0)$, и

$$
A=\lim _{z \rightarrow E_{0, \vartheta}}\left(E_{0, \vartheta}-z\right) \mathscr{M}_{0, \vartheta}(z)=\frac{\pi\left|E_{0, \vartheta}\right|}{2 \sin ^{2} \vartheta} .
$$

Поэтому из (55) вытекает, что мера $\sigma_{0, \vartheta}$ равна $\pi A \delta_{E_{0, \vartheta}}$ на $(-\infty, 0)$. Таким образом, $\sigma_{0, \vartheta}$ совпадает с $\mathcal{V}_{0, \vartheta}$ на $(-\infty, 0)$ для всех $\vartheta$.

ДОКАЗАТЕЛЬСТвО ТЕОРЕмЫ 3. Из утверждения 2 предложения 14 и леммы 22 следует, что оператор $U_{\kappa, \vartheta}$ существует и совпадает со спектральным преобразованием для $\mathfrak{t}_{\kappa, \vartheta}$. Поэтому согласно утверждению 3 предложения 14 и лемме 22 оператор $h_{\kappa, \vartheta}$ равен $L_{q}\left(X^{\mathfrak{t}_{\kappa, \vartheta}} \cap \mathcal{D}_{q_{\kappa}}^{\mathrm{r}}\right)$. В силу утверждения 1 предложения 8 и формулы (36) заключаем, что $h_{\kappa, \vartheta}$ есть самосопряженное расширение $h_{\kappa}$. В силу леммы 13 и формулы (32) имеем

$$
h_{\kappa, \vartheta}=L_{q_{\kappa}}^{u_{\vartheta}^{\kappa}(0)}
$$

В силу (50) и (52) каждая вещественная функция $f \in \mathcal{D}$, удовлетворяющая уравнению $l_{q_{\kappa}} f=0$, пропорциональна $u_{\vartheta}^{\kappa}(0)$ для некоторого $\vartheta \in \mathbb{R}$. Поэтому из леммы 11 и формул (36) и (57) следует, что каждое самосопряженное расширение $h_{\kappa}$ равно $h_{\kappa, \vartheta}$ для некоторого $\vartheta \in \mathbb{R}$. Пусть $\vartheta, \vartheta^{\prime} \in \mathbb{R}$. Согласно лемме 11 и формуле $(57)$ равенство $h_{\kappa, \vartheta}=h_{\kappa, \vartheta^{\prime}}$ справедливо тогда и только тогда, когда $u_{\vartheta}^{\kappa}(0)=c u_{\vartheta^{\prime}}^{\kappa}(0)$ для некоторого вещественного $c \neq 0$. Ввиду (50) и (52) последнее условие выполнено в том и только том случае, если $\vartheta-\vartheta^{\prime} \in \pi \mathbb{Z}$.

ЗАмечАниЕ 23. Следует отметить, что функция $q_{\kappa}$, задаваемая формулой (5), вещественна не только при вещественных $\kappa$, но и при чисто мнимых $\kappa$. Полное описание разложений по собственным функциям для этого случая приведено в работе [9]. Легко видеть, что $\mathfrak{t}_{\kappa, \vartheta}=\left(q_{\kappa}, \mathcal{D}_{q_{\kappa}}^{\mathrm{r}}, u_{\vartheta}^{\kappa}\right)$ является тройкой разложения и для мнимых $\kappa$, а спектральную меру для $\mathfrak{t}_{\kappa, \vartheta}$ по-прежнему можно вычислить, пользуясь формулами (55) и (56). Действуя таким образом, можно получить аналог теоремы 3 для мнимых $\kappa$.

\section{4. НЕПРЕРЫВНОСТЬ СПЕКТРАЛЬНЫХ МЕР}

В данном разделе мы докажем теорему 4.

Определим непрерывную функцию $\Phi$ на $(-1,1) \times \mathbb{R}_{+}$, полагая

$$
\Phi(\kappa, E)=-\frac{\ln E}{\pi \operatorname{sinc}(\pi \kappa)} \operatorname{sinc}\left(\frac{i \kappa}{2} \ln E\right),
$$

где целая функция sinc задается равенством

$$
\operatorname{sinc} \zeta= \begin{cases}\zeta^{-1} \sin \zeta, & \zeta \in \mathbb{C} \backslash\{0\}, \\ 1, & \zeta=0 .\end{cases}
$$

Имеем

$$
\Phi(\kappa, E)= \begin{cases}-\ln E / \pi, & \kappa=0, \\ (\sin \pi \kappa)^{-1}\left(E^{-\kappa / 2}-E^{\kappa / 2}\right), & 0<|\kappa|<1 .\end{cases}
$$

Для каждых $\vartheta \in \mathbb{R}$ и $-1<\kappa<1$ определим функцию $t_{\kappa, \vartheta}$ на $\mathbb{R}_{+}$формулой

$$
t_{\kappa, \vartheta}(E)=2+\Phi(\kappa, E)^{2}(1-\cos 2 \vartheta \cos \pi \kappa)+\Phi(\kappa, E)\left(E^{-\kappa / 2}+E^{\kappa / 2}\right) \sin 2 \vartheta .
$$


Прямое вычисление с использованием формул (17), (21) и (59) дает

$$
d \widetilde{\mathcal{V}}_{\kappa, \vartheta}(E)=t_{\kappa, \vartheta}(E)^{-1} \Theta(E) d E
$$

для всех $\vartheta \in \mathbb{R}$ и $-1<\kappa<1$. В силу неравенства Коши-Буняковского имеем

$$
|-c \cos 2 \vartheta+d \sin 2 \vartheta| \leqslant \sqrt{c^{2}+d^{2}}
$$

для любых $c, d \in \mathbb{R}$. Применяя эту оценку к $c=\Phi(\kappa, E)^{2} \cos \pi \kappa$ и

$$
d=\Phi(\kappa, E)\left(E^{-\kappa / 2}+E^{\kappa / 2}\right)=\Phi(\kappa, E) \sqrt{\Phi(\kappa, E)^{2} \sin ^{2} \pi \kappa+4},
$$

выводим из $(60)$, что $t_{\kappa, \vartheta}(E) \geqslant f\left(\Phi(\kappa, E)^{2}\right)$, где $f(y)=2+y-\sqrt{y^{2}+4 y}, y \geqslant 0$. Поскольку

$$
f(y)=\frac{4}{2+y+\sqrt{y^{2}+4 y}} \geqslant \frac{2}{2+y}, \quad y \geqslant 0,
$$

заключаем, что $t_{\kappa, \vartheta}(E)^{-1} \leqslant 1+\Phi(\kappa, E)^{2} / 2$ для всех $E>0,-1<\kappa<1$ и $\vartheta \in \mathbb{R}$. Из (58) вытекает, что $\kappa \rightarrow \Phi(\kappa, E)^{2}$ есть четная возрастающая на $[0,1)$ функция для любого $E>0$. Пусть $0<\alpha<1$. Ввиду (59) заключаем, что

$$
t_{\kappa, \vartheta}(E)^{-1} \leqslant 1+\frac{1}{2} \Phi(\alpha, E)^{2} \leqslant \frac{1}{2 \sin ^{2} \pi \alpha}\left(E^{\alpha}+E^{-\alpha}\right)
$$

для всех $E>0, \vartheta \in \mathbb{R}$ и $-\alpha \leqslant \kappa \leqslant \alpha$. Пусть $\varphi$ - ограниченная борелевская функция на $\mathbb{R}$ с компактным носителем и $B=(-1,1) \times \mathbb{R}$. Поскольку функция $(\kappa, \vartheta) \rightarrow t_{\kappa, \vartheta}(E)^{-1} \varphi(E)$ непрерывна на $B$ для любого $E>0$, из соотношений (61) и $(62)$ и теоремы о мажорированной сходимости следует, что функция $(\kappa, \vartheta) \rightarrow$ $\int \varphi(E) d \tilde{\mathcal{V}}_{\kappa, \vartheta}(E)$ непрерывна на $B$ и ограничена на $[-\alpha, \alpha] \times \mathbb{R}$ для любого $0 \leqslant \alpha<1$. Пусть

$$
B^{\prime}=\left\{(\kappa, \vartheta) \in B: \vartheta \in\left(\left|\vartheta_{\kappa}\right|, \pi-\left|\vartheta_{\kappa}\right|\right)+\pi \mathbb{Z}\right\} .
$$

В силу (16) и (19) имеем

$$
\int \varphi(E) d \mathcal{V}_{\kappa, \vartheta}(E)=\int \varphi(E) d \widetilde{\mathcal{V}}_{\kappa, \vartheta}(E)+b_{\varphi}(\kappa, \vartheta),
$$

где функция $b_{\varphi}$ на $B$ определяется соотношением

$$
b_{\varphi}(\kappa, \vartheta)= \begin{cases}\widetilde{\Phi}\left(\kappa,\left|E_{\kappa, \vartheta}\right|\right) \varphi\left(E_{\kappa, \vartheta}\right), & (\kappa, \vartheta) \in B^{\prime} \\ 0, & (\kappa, \vartheta) \in B \backslash B^{\prime}\end{cases}
$$

и непрерывная функция $\widetilde{\Phi}$ на $(-1,1) \times \mathbb{R}_{+}$задается равенством

$$
\widetilde{\Phi}(\kappa, E)=\frac{1}{2} E \pi^{2} \operatorname{sinc}(\pi \kappa)\left(\Phi(\kappa, E)^{2}+\frac{1}{\cos ^{2} \vartheta_{\kappa}}\right) .
$$

Для любого $(\kappa, \vartheta) \in B^{\prime}$ имеем $\left|\operatorname{ctg} \vartheta \operatorname{tg} \vartheta_{\kappa}\right|<1$, и из (18) и (20) следует, что

$$
E_{\kappa, \vartheta}=-\exp \left[\frac{\pi \operatorname{ctg} \vartheta}{2 \cos \vartheta_{\kappa}} \operatorname{sinc}\left(\vartheta_{\kappa}\right) g\left(\operatorname{ctg} \vartheta \operatorname{tg} \vartheta_{\kappa}\right)\right],
$$


где $g$ - такая непрерывная функция на $(-1,1)$, что $g(y)=y^{-1} \ln \left((1+y)(1-y)^{-1}\right)$ при $y \neq 0$ и $g(0)=2$. Таким образом, функция $(\kappa, \vartheta) \rightarrow E_{\kappa, \vartheta}$ непрерывна на $B^{\prime}$, и, следовательно, $b_{\varphi}$ есть борелевская функция на $B$. Оценивая $\Phi(\kappa, E)^{2}$, как и выше, получаем

$$
\widetilde{\Phi}(\kappa, E) \leqslant \frac{\pi^{2} E}{2 \sin ^{2} \pi \alpha}\left(E^{\alpha}+E^{-\alpha}\right), \quad(\kappa, E) \in[-\alpha, \alpha] \times \mathbb{R}_{+},
$$

для любого $0<\alpha<1$. С учетом (63) отсюда вытекает, что $b_{\varphi}$ ограничена на $[-\alpha, \alpha] \times \mathbb{R}$ для любого $0 \leqslant \alpha<1$. Для завершения доказательства нам осталось установить, что непрерывность $\varphi$ влечет непрерывность $b_{\varphi}$ на $B$. Пусть $-1<\kappa<1$. Из (18) и (20) следует, что $\left|E_{\kappa, \vartheta}\right|$ строго убывает от $\infty$ до 0 , когда $\vartheta$ пробегает от $\left|\vartheta_{\kappa}\right|$ до $\pi-\left|\vartheta_{\kappa}\right|$. Следовательно, для каждого $E>0$ имеется единственное число $\tau_{E}(\kappa) \in\left(\left|\vartheta_{\kappa}\right|, \pi-\left|\vartheta_{\kappa}\right|\right)$ такое, что $\left|E_{\kappa, \tau_{E}(\kappa)}\right|=E$. Ввиду непрерывности $E_{\kappa, \vartheta}$ по $(\kappa, \vartheta)$ функция $\tau_{E}$ непрерывна на $(-1,1)$ для любого $E>0$. Пусть $\beta>0$ таково, что $\varphi(E)=0$ для любого $E \leqslant-\beta$. Для $0<\alpha<1$ и $0<\delta$ определим открытое подмножество $B_{\alpha, \delta}$ множества $B$, полагая

$$
B_{\alpha, \delta}=\left\{(\kappa, \vartheta) \in(-\alpha, \alpha) \times \mathbb{R}: \vartheta \in\left(\tau_{\delta}(\kappa)-\pi, \tau_{\beta}(\kappa)\right)+\pi \mathbb{Z}\right\} .
$$

Если $\delta<1$, то в силу (63) и (64) имеем

$$
\left|b_{\varphi}(\kappa, \vartheta)\right| \leqslant \frac{\pi^{2} \delta^{1-\alpha}}{\sin ^{2} \pi \alpha} \sup _{E \in \mathbb{R}}|\varphi(E)|, \quad(\kappa, \vartheta) \in B_{\alpha, \delta} .
$$

Пусть $(\kappa, \vartheta) \in B \backslash B^{\prime}$ и $\varepsilon>0$. Выберем произвольное $\alpha \in(|\kappa|, 1)$ и найдем столь малое $\delta>0$, чтобы правая часть последнего неравенства была меньше $\varepsilon$. Тогда $B_{\alpha, \delta}$ является окрестностью $(\kappa, \vartheta)$, где абсолютное значение $b_{\varphi}$ меньше, чем $\varepsilon$. Тем самым доказано, что функция $b_{\varphi}$ непрерывна в каждой точке множества $B \backslash B^{\prime}$. Так как функция $b_{\varphi}$, очевидно, непрерывна на $B^{\prime}$, то теорема доказана.

\section{Доказательство леммы 17}

\section{ПРИЛОЖЕНИЕ А}

Пусть Ln - ветвь логарифма в $\mathbb{C}_{\pi}$, удовлетворяющая условию $\operatorname{Ln} 1=0$, и $p-$ аналитическая функция в $\mathbb{C} \times \mathbb{C}_{\pi}$, определенная соотношением $p(\kappa, r)=e^{\kappa \operatorname{Ln} r}($ так что $p(\kappa, r)=r^{\kappa}$ при $\left.r \in \mathbb{R}_{+}\right)$. Пусть $G$ - такая аналитическая функция в $\mathbb{C} \times \mathbb{C} \times \mathbb{C}_{\pi}$, что $G(\kappa, z, r)=p(1 / 2+\kappa, r) \mathcal{X}_{\kappa}\left(r^{2} z\right)$ для всех $\kappa, z \in \mathbb{C}$ и $r \in \mathbb{C}_{\pi}$. Тогда $G(\kappa, z, r)=$ $u^{\kappa}(z \mid r)$ для всех $\kappa, z \in \mathbb{C}$ и $r \in \mathbb{R}_{+}$. Определим функцию $F$ на $\mathcal{O} \times \mathbb{C} \times \mathbb{C} \times \mathbb{C}_{\pi}$, полагая

$$
\begin{aligned}
& F(\kappa, \vartheta, z, r)=\frac{G(\kappa, z, r) \sin \left(\vartheta+\vartheta_{\kappa}\right)-G(-\kappa, z, r) \sin \left(\vartheta-\vartheta_{\kappa}\right)}{\sin \pi \kappa}, \quad \kappa \in \mathcal{O} \backslash\{0\}, \\
& F(0, \vartheta, z, r)=G(0, z, r) \cos \vartheta+\frac{2}{\pi}\left[\left(\operatorname{Ln} \frac{r}{2}+\gamma\right) G(0, z, r)-p\left(\frac{1}{2}, r\right) \mathcal{Y}\left(z r^{2}\right)\right] \sin \vartheta
\end{aligned}
$$

для любых $z, \vartheta \in \mathbb{C}$ и $r \in \mathbb{C}_{\pi}$. Из (12), (13) и определения $F$ немедленно следует, что $F(\kappa, \vartheta, z, r)=u_{\vartheta}^{\kappa}(z \mid r)$ для любых $\vartheta, z \in \mathbb{C}, \kappa \in \mathcal{O}$ и $r \in \mathbb{R}_{+}$. Функция $(\vartheta, z, r) \rightarrow F(\kappa, \vartheta, z, r)$, очевидно, аналитична в $\mathbb{C} \times \mathbb{C} \times \mathbb{C}_{\pi}$ для любого фиксированного $\kappa \in \mathcal{O}$. Функция $\kappa \rightarrow F(\kappa, \vartheta, z, r)$ аналитична в $\mathcal{O} \backslash\{0\}$ и непрерывна при $\kappa=0$ 
(это доказывается тем же вычислением, которое было использовано для нахождения предела в (13)) и, следовательно, аналитична в $\mathcal{O}$ для любых фиксированных $\vartheta, z \in \mathbb{C}$ и $r \in \mathbb{C}_{\pi}$. Поэтому функция $F$ аналитична в $\mathcal{O} \times \mathbb{C} \times \mathbb{C} \times \mathbb{C}_{\pi}$ в силу теоремы Гартогса.

\section{ПРИЛОЖЕНИЕ Б}

\section{Доказательство леммы 17}

Пусть $T=L_{q}\left(C_{0}^{\infty}(a, b)\right)$. Так как оператор $L_{q}$ замкнут, то достаточно показать, что $T^{*}=L_{q}^{*}$. Для этого нам надо лишь проверить, что $D_{T^{*}} \subset D_{L_{q}^{*}}$, поскольку $L_{q}$ есть расширение $T$ и, следовательно, $T^{*}$ есть расширение $L_{q}^{*}$. В силу $(30) D_{L_{q}^{*}}$ состоит из всех элементов $[g]$, где $g \in \mathcal{D}_{q}$. Таким образом, для каждого $\phi \in D_{T^{*}}$ нам надо найти такую функцию $g \in \mathcal{D}_{q}$, что $\phi=[g]$. Пусть $\psi=T^{*} \phi$. Тогда

$$
\langle T[f], \phi\rangle=\langle[f], \psi\rangle, \quad f \in C_{0}^{\infty}(a, b) .
$$

Так как $(T[f])(r)=-f^{\prime \prime}(r)+q(r) f(r)$ для $\lambda$-п.в. $r \in(a, b)$, то

$$
-\int_{a}^{b} \overline{f^{\prime \prime}(r)} \phi(r) d r=\int_{a}^{b} \overline{f(r)}(\psi(r)-q(r) \phi(r)) d r, \quad f \in C_{0}^{\infty}(a, b) .
$$

Поскольку функции $q$ и $\phi$ локально квадратично-интегрируемы на $(a, b)$, функция $q \phi$ локально интегрируема на $(a, b)$. Выберем $c \in(a, b)$ и определим $h \in \mathcal{D}$, полагая

$$
h(r)=\int_{c}^{r} d \rho \int_{c}^{\rho}(\psi(t)-q(t) \phi(t)) d t .
$$

Очевидно, имеем $h^{\prime \prime}(r)=\psi(r)-q(r) \phi(r)$ для $\lambda$-п.в. $r \in(a, b)$. Интегрируя по частям, получаем

$$
\int_{a}^{b} \overline{f(r)}(\psi(r)-q(r) \phi(r)) d r=\int_{a}^{b} \overline{f^{\prime \prime}(r)} h(r) d r
$$

и, следовательно,

$$
\int_{a}^{b} \overline{f^{\prime \prime}(r)}(\phi(r)+h(r)) d r=0, \quad f \in C_{0}^{\infty}(a, b) .
$$

Это означает, что вторая производная $\phi+h$ в смысле обобщенных функций равна нулю. Поэтому найдутся такие $A, B \in \mathbb{C}$, что $\phi(r)+h(r)=A r+B$ для $\lambda$-п.в. $r \in(a, b)$. Определим $g \in \mathcal{D}$ формулой $g(r)=A r+B-h(r), r \in(a, b)$. Тогда, очевидно, имеем $[g]=\phi$. Так как $g^{\prime \prime}(r)=-\psi(r)+q(r) \phi(r)$, то $\left(l_{q} g\right)(r)=\psi(r)-q(r) \phi(r)+q(r) g(r)=$ $\psi(r)$ для $\lambda$-п.в. $r \in(a, b)$ и, следовательно, $l_{q} g=\psi$. Поэтому обе функции $g$ и $l_{q} g$ квадратично-интегрируемы, а значит, $g \in \mathcal{D}_{q}$.

Благодарности. Автор признателен Б. Л. Воронову и И. В. Тютину за полезные обсуждения.

\section{Список литературы}

[1] H. Hankel, Math. Ann., 8:4 (1875), 471-494.

[2] H. Weyl, Math. Ann., 68:2 (1910), 220-269.

[3] H. Weyl, Gött. Nachr., 1910 (1910), 442-467. 
[4] E. C. Titchmarsh, Eigenfunction Expansions Associated with Second-Order Differential Equations. Part I, Clarendon Press, Oxford, 1962.

[5] М. А. Наймарк, Линейные дифференциальные операторы, Наука, М., 1969.

[6] F. Gesztesy, M. Zinchenko, Math. Nachr., 279:9-10 (2006), 1041-1082, arXiv: math/ 0505120 .

[7] C. Fulton, Math. Nachr., 281:10 (2008), 1418-1475.

[8] A. Kostenko, A. Sakhnovich, G. Teschl, Internat. Math. Res. Notices, 2012:8 (2012), 1699-1747, arXiv: 1007.0136.

[9] D. M. Gitman, I. V. Tyutin, B. L. Voronov, J. Phys. A: Math. Theor., 43:14 (2010), 145205, 34 pp., arXiv: 0903.5277.

[10] A. G. Smirnov, J. Math. Phys., 56:12 (2015), 122101, 50 pp., arXiv: 1411.5351.

[11] W. N. Everitt, H. Kalf, J. Comput. Appl. Math., 208:1 (2007), 3-19.

[12] A. Erdélyi, W. Magnus, F. Oberhettinger, F. G. Tricomi, Higher Transcendental Functions, v. I, II, McGraw-Hill, New York-Toronto-London, 1953.

[13] K. Kodaira, Amer. J. Math., 71:4 (1949), 921-945.

[14] G. Teschl, Mathematical Methods in Quantum Mechanics, Graduate Studies in Mathematics, 99, AMS, Providence, RI, 2009.

[15] J. Weidmann, Spectral Theory of Ordinary Differential Operators, Lecture Notes in Mathematics, 1258, Springer, Berlin, 1987.

[16] C. Bennewitz, W. N. Everitt, "The Titchmarsh-Weyl eigenfunction expansion theorem for Sturm-Liouville differential equations", Sturm-Liouville Theory (Geneva, Italy, September 15-19, 2003), eds. W. O. Amrein, A. M. Hinz, D. B. Pearson, Birkhäuser, Basel, 2005, $137-171$.

Поступила в редакцию 24.08.2015, после доработки 9.11.2015 